\title{
Comparative genomic analysis of skin and soft tissue Streptococcus pyogenes isolates from low- and high-income settings
}

Saikou Y. Bah ${ }^{1,3 \#}$, Alexander J. Keeley ${ }^{2}$, Edwin P. Armitage ${ }^{3}$, Henna Khalid ${ }^{1}$, Roy R. Chaudhuri ${ }^{1}$ Elina Senghore ${ }^{3}$, Jarra Manneh ${ }^{3}$, Lisa Tilley ${ }^{4}$, Michael Marks ${ }^{5,6}$, Saffiatou Darboe ${ }^{3}$, Abdul K. Sesay ${ }^{3}$, Thushan I de Silva ${ }^{2,3} \&$ Claire E. Turner ${ }^{1 \#}$, and on behalf of The MRCG StrepA Study Group

1. Department of Molecular Biology \& Biotechnology, The Florey Institute, University of Sheffield, Sheffield, United Kingdom.

2. Department of Infection, Immunity \& Cardiovascular Disease, The Florey Institute, University of Sheffield Medical school, Sheffield, United Kingdom.

3. The Medical Research Council, The Gambia at the London School of Hygiene and Tropical Medicine, The Gambia.

4. Department of Microbiology, Sheffield Teaching Hospitals NHS Foundation Trust, Sheffield, United Kingdom

5. Clinical Research Department, Faculty of Infectious and Tropical Diseases, London School of Hygiene \& Tropical Medicine, London, United Kingdom

6. Hospital for Tropical Diseases, London, United Kingdom

\section{\#Joint Corresponding authors:}

Dr Saikou Bah

saikouybah@gmail.com

Dr Claire Turner

c.e.turner@sheffield.ac.uk 


\section{Abstract}

27 Streptococcus pyogenes is a leading cause of human morbidity and mortality, especially in resource limited settings. The World Health Organisation has recently made a vaccine for $S$. pyogenes a global health priority to reduce the burden of the post-infection rheumatic heart disease. For a vaccine to be active against all relevant strains in each region, molecular characterisation of circulating $S$. pyogenes isolates is needed. We performed extensive comparative whole genome analyses of $S$. pyogenes isolates from skin and soft tissue infections in The Gambia, West Africa, where there is a high burden of such infections. To act as a comparator to this low-income country (LIC) collection of isolates, we performed genome sequencing of isolates from skin infections in Sheffield, UK, as representative high-income country (HIC) isolates. LIC isolates from The Gambia were genetically more diverse (46 emmtypes in 107 isolates) compared to HIC isolates from Sheffield (23 emm-types in 142 isolates), with only 7 overlapping emm-types and with diverse genetic backgrounds. Characterisation of other molecular markers indicated some shared features, including a high prevalence of the skin infection-associated emm-pattern D and the variable fibronectin-collagen-T antigen (FCT) types FCT-3 and FCT-4. A previously unidentified FCT (FCT-10) was identified in the LIC isolates, belonging to two different emm-types. A high proportion $(79 / 107 ; 73.8 \%)$ of LIC isolates carried genes for tetracycline resistance, compared to 53/142 (37.3\%) HIC isolates. There was also evidence of different circulating prophages, as very few prophage-associated DNases and lower numbers of superantigens were detected in LIC isolates. Our study provides much needed insight into the genetics of circulating isolates in a LIC (The Gambia), and how they differ from those circulating in HICs (Sheffield, UK). Common molecular features may act as bacterial drivers for specific infection types, regardless of the diverse genetic background. 
50

51

52

53

54

55

56

57

\section{Introduction}

Streptococcus pyogenes (Group A Streptococcus, GAS) is a human-specific pathogen and a leading cause of morbidity and mortality, especially in resource-limited countries. S. pyogenes can cause diseases ranging from mild superficial infections, such as impetigo and pharyngitis, to invasive diseases such necrotising fasciitis and streptococcal toxic shock syndrome (1) and can also cause post-infection autoimmune sequalae such as acute rheumatic fever (ARF) leading to rheumatic heart disease (RHD). There is a substantial global burden of RHD, accounting for approximately 320,000 deaths in 2015 , the majority of which were recorded in sub-Saharan Africa (2). Recognising this burden, the World Health Organisation (WHO) has prioritised the need for a vaccine that would have global coverage, and recommended an increase in research, especially in low- to middle-income countries (LMICs) (3).

Progress towards a vaccine for S. pyogenes has been hampered over the years by the association of the most promising vaccine candidate, the surface protein M, with the development of RHD. This may be circumvented by targeting the $\mathrm{N}$ terminal portion of the $\mathrm{M}$ protein, but this region is hypervariable, thus any vaccine would be serotype/genotype specific. S. pyogenes isolates are genotyped by sequencing the corresponding hypervariable $5^{\prime}$ region of the $\mathrm{M}$ proteinencoding gene, emm. Over 220 different emm-types have been identified globally, but in high income countries (HICs) the majority of disease is caused by a limited number of emm-types, with emm 1 being the most common. A 30-valent M-protein vaccine has been developed and is undergoing clinical trials but is based on genotypes predominantly circulating in Europe and North America $(4,5)$. The limited available data for S. pyogenes in LMICs suggest a far more genetically diverse population than that seen in HICs (6-9). More extensive, global genomic analysis may reveal another vaccine target or combination of targets that would be applicable in these settings. 
74 The emm gene lies within the $S$. pyogenes core $m g a$ (multi gene activator) regulon locus, and upstream and/or downstream of the $\mathrm{emm}$ gene there may be additional $\mathrm{emm}$-like genes. There have been ten different emm patterns identified, based on the genes within the mga regulon, which form three main groupings: A-C, D or E. The majority of emm types have been associated with only one emm pattern (10). There is some epidemiological evidence supporting the existence of tissue tropism among emm types, with preference for either pharyngeal or skin infection sites, or "generalists" that are equally able to infect both sites (11). There is also an association with this tissue tropism to emm pattern; pharyngeal specialists are pattern A-C, the skin specialists are pattern D, and the generalists are pattern E (10). However, much of this evidence comes from population-based surveys where there is greater sampling of pharyngeal infections in HICs but more skin infections (impetigo/pyoderma) in LMICs (12). Whether this reflects an actual difference in the prevalence of infection types is unclear, as data is lacking for both skin infections in HICs and pharyngeal infections in LMICs (12-14).

It is estimated that more than 162 million children have impetigo/pyoderma at any given time, predominantly in LMICs, although data for Europe, South-East Asia and North America is very limited (14). A recent study in The Gambia, West Africa, identified a 17.4\% prevalence of pyoderma in children, with S. pyogenes as a leading infection cause (15). This was higher than the estimated global prevalence of $12.3 \%$ (14). The association with scabies infestation in The Gambia was lower than that seen in other settings, but there was an increase in pyoderma prevalence from $8.9 \%$ to $23.1 \%$ during the rainy season (15). Whilst there may be environmental and socio-demographic factors underpinning the high burden of pyoderma in The Gambia, there may also be bacterial factors involved and potentially tissue tropism. To investigate this, and to provide molecular characterisation of $S$. pyogenes causing skin infections in The Gambia, we performed whole genome sequencing on the isolates obtained from our previous study (15). To act as a comparative HIC collection of isolates, we also 
performed whole genome sequencing and molecular characterisation of $S$. pyogenes isolated from skin infections in Sheffield, UK. Our study highlights the genetic diversity observed in isolates, including emm-pattern, emm-cluster and FCT-type, supporting the hypothesis that there are bacterial factors driving certain types of infection.

\section{Material and Methods}

\section{Isolates}

S. pyogenes skin pyoderma lesion isolates from one hundred and thirty-six children under the age of 5 in the peri-urban setting of Sukuta in The Gambia, collected between May and

109 September 2018 (15), were available for whole genome sequencing. As previously described, swabs were stored in liquid Amies transport medium before being taken to Medical Research

111 Council Unit The Gambia at London School of Hygiene \& Tropical Medicine (MRCG at

112 LSHTM) for culture and identification of $S$. pyogenes (15). To provide a representative 113 collection of $S$. pyogenes from a HIC for comparison, 160 sequentially cultured skin and soft 114 tissue infection (SSTI) isolates were collected from the Department of Laboratory Medicine,

115 Northern General Hospital, Sheffield, UK between January and April 2019. No patient data were obtained for these isolates so no selection was applied for patient characteristics such as

117 age or sex.

\section{Whole genome sequencing}

119 Streptococcal DNA was extracted from isolates using a method previously described (16). For

120 Gambian isolate DNA, sequencing libraries were prepared using the NEBNext Ultra ${ }^{\mathrm{TM}}$ II DNA

121 Library Prep Kit for Illumina and sequenced on an Illumina MiSeq at MRCG. The MiSeq V3 reagent kit was used to generate $250 \mathrm{bp}$ paired end reads following the Illumina recommended 
denaturation and loading recommendations which included a 5\% PhiX spike-in. Raw sequence

assessment was

performed

using FastQC

(v0.11.8;

125

126

127

128

129

130

131

132

133

134

135

136

137

138

139

140

141

142

143

144

145

146

147

https://www.bioinformatics.babraham.ac.uk/projects/fastqc) with default settings and reads were trimmed using Trimmomatic (v0.38) with following settings: LEADING:3 TRAILING:3

SLIDINGWINDOW:4:15 MINLEN:36 (17). Sequencing of the genomic DNA from Sheffield, UK collection isolates and a selection of isolates from the Gambia collection that were subjected to repeat sequencing after failing quality control, was provided by MicrobesNG (microbesng.com) using the Nextera XT Library Prep kit (Illumina) and the Illumina HiSeq/NovaSeq platform generating 250bp pair end reads. Data was subjected to MicrobesNG quality control and Trimmomatic pipelines. Short read sequence data were submitted to the sequence read archive and accession numbers are provided in Supplementary Table 1.

\section{Whole genome sequence analysis}

De novo assembly was performed using SPAdes (v3.13.1) with k-mers sizes of 21, 33, 55 and 77 (18). Assembly qualities statistics were generated using Quast (19) (Supplementary Table 1) and any assemblies with more than 500 contigs and a total genome size greater than $2.2 \mathrm{Mb}$ were removed from downstream analyses. Prokka (v1.13.3) was then used to annotate the assemblies (20) and the pangenome determined using Roary (v3.12.0) with a 95\% identity level (21). Single nucleotide polymorphism (SNP) distances were determined from the Roary coregene alignment output using snp-dists (v0.7.0, https://github.com/tseemann/snp-dists). RAxML (v8.2.12) (22) was used to generate maximum likelihood phylogenetic trees based on the core-gene alignment, with GTR substitution model and 100 bootstraps. Phylogenetic trees were visualized and annotated using iTOL (23). The emm types were determined from the $d e$
novo
assemblies
using
emm_typer.pl

(github.com/BenJamesMetcalf/GAS_Scripts_Reference). Where necessary, emm genes were manually located and type determined using the CDC emm-typing database 
148 (www.cdc.gov/streplab). New emm-subtypes were submitted to the database for assignment.

149 Multi-locus sequence types (MLSTs) were determined using the MLST database

150 (pubmlst.org/spyogenes) and a script from the Sanger pathogen genomics group

151 (github.com/sanger-pathogens/mlst_check). Any new alleles and sequence types were

152 submitted to the PubMLST database.

\section{Variable factor typing}

154 The presence of superantigens; speA, speC, speG, speH, speI, speJ, speK, speL, speM, speQ, speR, ssa and smeZ, and DNases; sdal, sda2, sdn, spdl, spd3 and spd4 were initially determined by BLAST of representative gene sequences against the de novo assemblies. Gene presence was then additionally confirmed by BWA-MEM (24) mapping of the short read sequences to a pseudo sequence of concatenated superantigen and DNase genes; coverage of

159 at least 10 reads across the whole gene was used to confirm presence. Where the BLAST and mapping did not agree, results were manually inspected in the annotated de novo assemblies. (github.com/tseemann/abricate) using the ARG-ANNOT database (25), setting a minimum coverage of $70 \%$ and percentage identity of $75 \%$.

164 The nucleotide sequences for $\operatorname{cov} R, \operatorname{cov} S$ and $\operatorname{roc} A$ regulatory genes and the has $A$, has $B$ and

165 has $C$ capsule biosynthesis genes from the $S$. pyogenes $\mathrm{H} 293$ reference genome (emm89, 166 NZ_HG316453.1) were used as queries in blastn searches against the de novo assemblies. The

167 start and end coordinates of the best BLAST hits were converted into BED files and used to 168 extract the nucleotide sequences from the de novo assemblies using BEDTools (v2.27.1) (26).

169 Extracted gene sequences were then translated into amino acids and variants determined in 170 comparison to the corresponding amino acid sequences of the reference (H293) protein 
171 sequences. For has $A B C$, only nonsense variants and gene absence were recorded

172 (Supplementary Table 1).

\section{Emm pattern and FCT regions}

174 To determine the emm pattern in the genome of each isolate, in silico PCR

175 (https://github.com/simonrharris/in_silico_pcr) was used to extract the sequence of the whole

$176 m g a$ regulon (the beginning of $m g a$ to the end of $s c p A$ ) from de novo assemblies and then

177 annotated with Prokka. To improve assemblies where the mga regulon was not within

178 contiguous sequence, de novo assemblies were ordered against a completed reference genome

179 of the same emm-type (where available) using ABACAS (27) and the in silico PCR repeated.

180 An emm pattern of I, II, III, IV, V or VI was assigned using BLAST to identify genes followed

181 by visual determination of gene location within the regulon. For 22 LIC and 3 HIC isolates,

182 the emm pattern could not be determined as contiguous sequence for the mga regulon could not be obtained (detailed in Supplementary Table 1).

184 Alleles of the emm-like genes enn and mrp were assigned by comparison to those identified by

185 Frost et al. (28), ensuring 100\% nucleotide identity across the entire gene sequence. Where we

186 could not obtain contiguous sequence for the mga regulon, enn and mrp alleles were

187 determined by BLAST of each allele sequence against the entire de novo assembly. New alleles

188 for enn and mrp were kindly assigned by Prof Pierre Smeesters and Dr Anne Botteaux. In some

189 cases, breaks in the de novo assemblies occurred within the enn gene and therefore alleles could

190 not be confirmed (detailed in Supplementary Table 1).

191 To determine the arrangement of the genes in the FCT region and the FCT type, in silico PCR

192 was used to extract the FCT region and annotated with Prokka. Assemblies in which amplicons

193 were not obtained due to contig break in the FCT regions, were again ordered against a close reference of the same emm-type (where available). The ORFs within each extracted FCT region 
195

196

197

198

199

200

201

202

203

204

205

206

207

208

209

210

211

212

213

214

215

were blasted against the entire NCBI database and, in combination with order of the genes, the FCT types were assigned based on previously assigned FCT type where possible (29). For some isolates, it was not possible to obtain contiguous sequence for the FCT region and so the FCT type was estimated based on manual inspection of the de novo assembly and identification of FCT associated genes through BLAST.

\section{Results}

\section{Genetic diversity of S. pyogenes LIC and HIC skin and soft tissue isolates}

We performed whole genome sequencing on 115 of 127 S. pyogenes skin infection isolates collected in The Gambia (15). After quality control and filtering of reads and de novo assemblies, we obtained high quality genome sequence data for a total of 107 Gambian (LIC) S. pyogenes isolates for further analyses (Supplementary Table 1). Within the genomes of these 107 isolates, we determined 46 different emm-types, with no obvious dominant emm-type; the most common being emm80 (6/107, 6\%), closely followed by emm85, emm229 and emm/stG1750 (5/107 isolates, 5\% each). Although emm/stG1750 has been previously identified in group G streptococci, in this case these isolates were $S$. pyogenes with the group A carbohydrate. The multi-locus sequence types (STs) for all 107 isolates were determined and revealed 57 different types, of which 25 were assigned for the first time. Although multiple STs could be found within single emm-types, no STs were shared by multiple emm-types.

An emm-pattern could be assigned to the majority of isolates using the previously determined classifications. The exceptions were two emm147 isolates, one emm162 isolate, one emm247 isolate and five $\mathrm{emm} / \mathrm{stG} 1750$ isolates, for which an $\mathrm{emm}$ pattern had not been previously described. For the 98 isolates with known emm-patterns, $48 \%(\mathrm{n}=47)$ were $\mathrm{D}, 40 \%$ were $\mathrm{E}$ $(\mathrm{n}=39)$ and $12 \%(\mathrm{n}=12)$ were A-C (Figure 1 and Figure 2). In addition to emm-pattern, an emmcluster type could also be assigned to these 98 isolates. The emm-cluster type is based on the 
sequence of the full $\mathrm{M}$ protein and is broadly associated with emm-pattern (30). The majority of isolates $(56 / 98, \sim 57 \%$,$) were assigned to one of the six E emm-cluster types: E1 (n=4), E2$ $(n=2), E 3(n=14), E 4(n=16), E 5(n=2), E 6(n=18)$, representing 25 emm-types. All E1-E4 and all but four E6 emm-types were positive for the serum opacity factor (sof) gene, commonly associated with E emm-clusters (11), however E5 emm-types were sof negative. The remaining isolates were A-C4 (n=6), D1 (n-1), D2 (n=1), D4 (n=17) or singletons $(n=17)$.

Phylogenetic analysis of the core genome of all 107 LIC isolates showed clustering by emmtype (Figure 1). The exceptions to this were emm25, emm65, emm85, emm89 and emm209, analysis identified a median of 22 SNPs when comparing isolates with the same emm type (range; 0-11,142 SNPs), and a median of 9,816 SNPs when comparing isolates with different emm types (range 1,423 to 12,428) (Supplementary Figure 1A).

After read quality filtering and assembly assessment, we obtained draft genomes from $142 S$. pyogenes skin infection isolates collected in Sheffield, UK. Within these 142 HIC isolates there were 23 different emm-types but $\sim 59 \%$ of the isolates were represented by just $5 \mathrm{emm}$ types: emm 108 (30/142, 21\%), emm89 (19/142, 13\%), emm12 (15/142, 11\%), emm1 (10/142, $7 \%)$ and emm4 (9/142, 6\%). An emm-pattern could be assigned to all 142 isolates and 36\% $(\mathrm{n}=51)$ were D, 35\% $(\mathrm{n}=50)$ were E and $29 \%(\mathrm{n}=41)$ were A-C (Figure 2 and Figure 3). An emm-cluster type was also assigned to all 142 isolates and the majority of isolates were D4 $(\mathrm{n}=50,35 \%)$. No other D cluster-types were found. The most common E cluster type was E4 $(n=26)$, followed by E6 $(n=14)$, E1 $(n=9)$ and E3 $(n=2)$ (Figure 2). The A-C clusters were 240 represented by emm $1(\mathrm{~A}-\mathrm{C} 3, \mathrm{n}=10)$, emm $12(\mathrm{~A}-\mathrm{C} 4, \mathrm{n}=15)$ and emm3 (A-C5, n=5), which were absent emm-types in the LIC population (Figure 2). Only emm5 (n=4) and emm6 $(\mathrm{n}=7)$ were singleton emm-cluster types. 
243 Consistent with the fewer emm-type within the HIC isolate collection, we identified only 28

244 different STs, the most common being ST14, ST101, ST36 and ST28, reflective of their

245 association with the dominant genotypes emm108, emm89, emm12 and emm1, respectively.

246 As with the LIC isolates, STs were unique to a single emm-type.

247 The phylogenetic analysis of the HIC isolates based on core-genome SNPs also grouped

248 isolates into lineages based on emm-types, and all emm-types formed single lineages (Figure

249 3). Pairwise genetic distance between isolates identified a median of 17 SNPs between isolates

250 of the same emm type (range 0 to 2206), compared to a median of 11100 SNPs distance

251 between isolates belonging to different emm types (range 3057 to 12339) (Supplementary

252 Figure 1B).

Surprisingly, only seven out of the 62 total emm-types identified were common to both LIC and HIC isolates: emm4, 28, 75, 77, 80, 81 and 89. However, except for emm80 (emm80.0), the other six overlapping emm-types were of different emm sub-types between the two sites (Figure 2, Supplementary table 1). All were emm-cluster E emm-types, except emm80 which belongs to $\mathrm{emm}$-cluster D4. Pairwise comparison of isolates from the two different sites within each of these emm-types revealed a level of genetic distance similar to that observed when isolates of different emm-types were compared, indicating that, although they may share an emm-type, they do not share a core genome.

It is also possible that closely related isolates may exist within both collections but carry different emm genes. Core-gene phylogeny of all isolates from both sites combined showed clear segregation of isolates from different sites, except in one instance where an emm 192 HIC isolate clustered with two emm56 LIC isolates (Supplementary Figure 2). genes. However, while 1416 genes were present in at least one HIC isolate and absent from all 
LIC isolates, 3418 genes were present in at least one LIC isolate but absent from all HIC isolates. This indicates a greater accessory genome in LIC isolates. The core genome of LIC isolates alone was 1288, similar to HIC isolates at 1242, but there was a total of 6408 genes in LIC isolates compared to 4411 genes in HIC isolates.

\section{The Mga-regulon diversity}

272 The core Mga-regulon includes the $m g a$ gene and all intervening genes up to and including scpA (encoding for the C5a peptidase). Genes within this region encode proteins involved in cell invasion and immune evasion and include those for the $\mathrm{M}$ protein, encoded by $\mathrm{emm}$, and the M-like proteins Mrp and Enn. The composition of the intervening genes that define the Mga-regulon, as well as the type of M protein and positivity for serum opacity factor (sof),

277 relates to the emm pattern (A-C, D or E) $(10,28)$. We were able to determine the composition of the Mga-regulon for 36/46 emm-types for 85/107 LIC isolates and all 23/23 emm-types for 139/142 HIC isolates. Among the LIC isolates, we could not confirm the Mga-regulon for all isolates within ten different emm types, because it was not contiguous in the de novo assemblies, possibly due to sequence quality or repetitive regions. For the HIC isolates, this was the case for only single isolates within emm types emm 1, 12 and 108, and other isolates within these emm-types had confirmed Mga-regulons.

Six different Mga-regulon compositions were identified across isolates from both sites (Figure 4) but the vast majority of emm-types from both sites were Mga-regulon type I, consisting of mga, mrp, emm, enn and scpA. This type was found in 31/36 emm-types in LIC isolates and isolates and HIC isolates, respectively. Mga-regulon type II, with the emm1 streptococcal inhibitor of complement (sic) or emm12 SIC related gene ( $d r s)$, was only found in HIC isolates. 
290 Alleles for $m r p$ and enn were extracted and compared for associations with emm and geographical location of the isolate. Ninety-seven mrp genes and 92 enn genes were extracted from the 107 LIC isolate genomes, resulting in 44 unique mrp sub-alleles and 48 unique enn sub-alleles. From the $142 \mathrm{HIC}$ isolate genomes, we extracted $101 \mathrm{mrp}$ genes and 99 enn genes, resulting in 22 unique $m r p$ sub-alleles and 21 unique enn sub-alleles. For the majority, unique alleles were associated with emm-type and geographical location, although phylogenetic analysis did show overall there was limited geographical restriction between closely related one clade associated with E cluster $\mathrm{emm}$-patterns while the other associated with a mix of $\mathrm{emm}$ patterns. We did identify some instances of the same mrp allele associated with different emm types, although, with one exception, this was restricted to the LIC isolates. The mrp202 allele was shared by emm 119 and $e m m 162$ isolates and $m r p 60$ was shared by emm 85 and $e m m 89$ isolates. Sub-alleles (same amino acid sequence but different nucleotide sequence) mrp193.14 and $m r p 193.15$ were found in emm116 and emm86, respectively. Different sub-alleles of mrp 195 were found in the LIC emm18, emm 95 and emm/stg1750 isolates but also in HIC emm53 isolates. A similar pattern was also found with enn, with different sub-alleles of enn199 found in the LIC emm65 and emm182 isolates, and sub-alleles of enn 26 found in the LIC emm168 but also HIC emm89 isolates.

308 We also looked for the presence of the fbaA gene, downstream of $s c p A$ (outside of the Mga309 regulon), which encodes a surface protein associated with the infection potential of pattern D 310 skin isolates $(11,31)$. This gene was found in all D pattern and E pattern isolates but was absent 311 in $75 \%$ of A-C pattern HIC and LIC isolates (Supplementary Table 1). 


\section{Diversity of superantigens and DNases in the skin isolates}

313 The complement of superantigen and DNase genes S. pyogenes isolates can carry varies,

314 mainly due to the association of these factors with mobile bacteriophages. There are potentially

31513 different superantigen genes that can be carried by S. pyogenes; speA, speC, speH, speI,

316 speK, speL, speM, and ssa are prophage-associated, while speG, speJ, speQ, speR and smeZ

317 are chromosomal. Of the 107 LIC isolates, 99 (93\%) carried spe G and 97 (91\%) had smeZ.

318 Less common were speJ and the co-transcribed speQ/speR, found in 43/107 (40\%) and 7/107

319 (7\%), isolates respectively (Figure 5). A similar pattern was observed in the HIC isolates, with

$320130 / 142(92 \%)$ and 134/142 (94\%) isolates carrying speG and smeZ respectively, while speJ

321 was present in 46/142 (32\%) and speQ/speR was carried in 9/142 (6\%) isolates.

322 Of the prophage-associated superantigens, speC was the most predominant in the LIC isolates, carried by 26/107 (24\%) isolates (Figure 5), and in the HIC isolates, although much higher at $55 \%$ (78/142). Two (out of eight) emm43 HIC isolates and the single emm102 HIC isolate each carried two copies of $s p e C$, as well as two copies of the associated DNase spdl. These appeared to be carried on two separate phages integrated at two different sites.

327 In the HIC isolates, prophage-associated ssa was present in 63/142 (44\%) isolates, compared to only $8 / 107(7 \%)$ of the LIC isolates.

Interestingly, speA was almost equally common in the LIC isolates $(22 / 107,21 \%)$ as in the

330 HIC isolates $(28 / 142,20 \%)$, but, apart from one emm89 isolate, all LIC isolates carried the speA4 allele (or a speA very close to this allele) which is $11 \%$ divergent from the other alleles

332 (32) and was associated with a prophage-like element rather than a full prophage. This 333 prophage-like element has been previously identified in the emm6 reference strain 334 MGAS10394, termed $\Phi 10394.2$, and comprised of transposases and fragments of speH and speI (Supplementary Figure 4) (32). Previously, it has only been found in emm6, emm32, 
emm67 and emm77. In the HIC isolate collection this element, and the speA4 allele, was only found in emm6. The only isolate in the LIC isolate collection that carried a different speA allele, did not share any substantial identity to other known prophages in S. pyogenes (determined by BLASTn against the entire NCBI database).

341 Prophage-associated speH, speI, speK, speL and speM were detected at fairly similar levels between the two sites; $15 \%, 13 \%, 20 \%, 5 \%$, and $7 \%$ respectively in LIC isolates compared to $25 \%, 16 \%, 25 \%, 8 \%$ and $8 \%$ in the HIC isolates (Figure 5). One LIC emm65 isolate had an apparent fusion gene comprised of 5' speK and 3' speM. An alignment of the 259 amino acids (aa) of this potential fusion protein showed $100 \%$ identity to the first 180 aa of SpeK and a $100 \%$ of the remaining 181-259 aa to the last 159-237 aa of SpeM (Supplementary Figure 5).

347 We also tested for the presence of the prophage-associated DNases $s d a$, $s d n$, $s p d 1$, spd3 and spd4 (33). Only two prophage-associated DNases were identified in the LIC isolates; spdl, 26/107 (24.3\%) and spd3, 2/107 (1\%). These were also the most prevalent in the HIC isolates, at 79/142 (56\%) and 99/142 (70\%), respectively but we also detected sdal; 7/142(5\%), sda2; 23/147(16\%), sdn 23/147(16\%) and spd4 10/142(7\%).

\section{Hyaluronic capsule biosynthesis genes}

353 Although the hyaluronic capsule is considered an important virulence factor, recently it was 354 shown that genotypes emm4, emm22 and emm89 lack the hasABC operon required to synthesise the capsule. Additionally, in HICs there is a high proportion of isolates within different genotypes whereby has $A$ or hasB has either been deleted or carries a mutation that would render the encoded protein non-functional, predicted to result in the lack or reduction of capsule (33). The hasABC operon was detected in all the LIC isolates, including the emm4 and emm89 isolates, supporting the findings that they have a different core genome compared to 
HIC emm4 and emm89, which all lacked the has $A B C$ operon. No variations were detected in the has $A$ and hasB genes that would lead to truncated proteins in the LIC isolates, except for isolates and consistent with previous findings (33), all emm28, emm77 and emm87 isolates were predicted to produce truncated HasA, and all emm81 and emm 94 predicted to produce truncated HasB. Three other isolates were predicted to produce truncated HasA and a further two to produce truncated HasB, but these were sporadic examples within emm-types (Supplementary Table 1).

\section{FCT-types in the LIC and HIC isolates}

The Fibrinogen collagen binding T-antigen (FCT) region, which is classified into 9 different types (FCT1-9), encodes for pilin structural and biosynthesis proteins and adhesins that could be potential determinants of genetics basis for tissue tropism (34). Therefore, we investigated the diversity of the FCT regions in isolates across the two geographical settings. Eight different patterns were identified across the two sites, corresponding to FCT1-6 and FCT9, as well as a previously unidentified pattern found among the LIC isolates, which we termed FCT10; it was similar to FCT5, but with an additional fibronectin binding protein (Figure 6). FCT3 was found in the most emm-types in both LIC and HIC isolate collections, 9/23 (39\%) and 20/46 (43\%), respectively, although this represented only $23 \%$ of the HIC isolates compared to $41 \%$ of LIC isolates. FCT4 was also found in a high proportion of emm types, accounting for 7/23 (30\%) and 11/46 (24\%) emm-types, representing $28 \%$ and $30 \%$ of HIC and LIC isolates, respectively.

380 Due to the prevalence of emm108 and emm 1 in HIC, 33\% of isolates were either FCT1 or 381 FCT2, whereas only $6 \%$ of the LIC isolates were FCT1 and no LIC isolates were FCT2. There was only one example of isolates of the same emm-type with two different FCT-types, and that was within the two LIC emm118 isolates. While one emm118 (ST1205) isolate was estimated 
385

386

387

388

389

390

391

392

393

394

395

396

397

398

399

400

401

402

403

404

405

406

407

isolates. The FCT regions in both emm 118 isolates however were estimated as they were not found within a single contiguous sequence.

We also compared the amino acid sequences of the FCT regulatory genes rofA, $n r a$ and $m s m R$ and identified a number of different of variations. For the majority, variations were common to all isolates within an emm-type and there were no obvious variations that may affect function. We found that 9/10 HIC emm1 isolates carried three variations within RofA that characterised them as being part of the $\mathrm{M}_{\mathrm{UK}}$ lineage associated with high speA expression (35). No other isolates were found to carry any of these three RofA variations.

\section{Prevalence of antimicrobial resistance genes}

Of the 107 Gambian assemblies, the tetM gene encoding for tetracycline resistance was identified in $79 / 107(73.8 \%)$, and 37 of these (33.6\% of the total population) also carried the tet $L$ gene and one carried tetK. Furthermore, $d f r G$ or $d f r K$, both encoding for trimethoprim resistance, were identified in 10/107 (9.3\%) and 17/107 (15.9\%) of isolates respectively. Only $53 / 142(37.3 \%)$ of the HIC isolates carried the tetM gene (Figure 1 and 3) and no other resistance genes were found except for ermA in 8/142 (6.5\%) isolates and two emm11 isolates carried ermB, sat4A and aph3.

\section{Vaccine antigen diversity}

Based on the number of isolates with emm-types present in the vaccine, the potential coverage of the 30 -valent M protein vaccine in the LIC isolates was $24 \%$, with only 11 vaccine-included emm-types (Supplementary Figure 6). On the other hand, the potential coverage of the HIC isolates was $61 \%$, although only 14 were vaccine-included emm-types. This suggests limited potential for this vaccine for low-income settings such as The Gambia, although there may be potential for cross-protection as has been seen for some emm-types $(4,36)$. 
408 Among other potential vaccine candidates, the genes spy0651, spy0762, spy0942, pulA, oppA, $409 s h r, s p e B$, adi, ropA(tf), spyCEP, slo, spyAD, fbp54 and scpA were recently highlighted as 410 conserved potential targets (37). All LIC and HIC isolates carried all 14 genes and BLASTp 411 indicated that all genes were highly conserved in all isolates with less than $1 \%$ sequence 412 divergence (>99\% identity) from the corresponding genes in reference genome MGAS5005 413 (emm1).

\section{Discussion}

415 The overall global burden of S. pyogenes infection and associated post-infection sequalae, 416 highlights the need for more research into treatment and prevention, with a particular focus on 417 vaccine development. Maximal global impact of a preventative vaccine against $S$. pyogenes 418 can only be achieved on the back of better understanding of the global diversity of the $S$. 419 pyogenes population, but to date, large-scale genomic studies have been mainly focused on 420 HIC isolates. The Gambia, West Africa is a LIC with a high burden of streptococcal skin 421 infections (15). Studies on circulating emm-types in this region, and in other African countries, 422 indicate a much higher level of diversity than that seen in HICs (6- 9) and this is reflected in 423 the limited African genomic data (37). In this study, we aimed to contribute genomic data and

424 provide molecular characterisation of S. pyogenes in The Gambia by whole genome sequencing 425 isolates collected during a population-based study of skin infections in children aged 5 years 426 and under. To act as a comparison isolate collection, we also genome sequenced isolates from 427 Sheffield, UK to represent HIC isolates.

428 Consistent with other findings from LICs (9), we identified a high number of different emm429 types in the LIC isolate collection from The Gambia compared to the HIC isolate collection 430 from the UK, and no dominant type. In the HIC isolates, five emm-types (emm108, emm89, emm12, emm 1 and emm4) accounted for $\sim 60 \%$ of the isolates. There was also limited overlap 
across the two sites with only 7 shared emm-types; emm4, 28, 75, 77, 80, 81 and 89 . However,

433 it was clear that these emm-types represented a different genetic background between the two

434 locations, supporting previous findings that emm might not be a good marker for characterising a diverse global population (37).

Although we did not specifically select for impetigo isolates or patient age range amongst the HIC isolate collection, all were associated with some form of non-invasive skin infection. Little molecular information is available for S. pyogenes causing skin infections in the UK, as isolates are not routinely collected and typed, or for other HICs. The dominant emm genotypes found in the HIC isolates reflected what has been found in other types of infections, with emm1, emm12 and emm89 leading among invasive isolates in the UK (33) and emm1, emm4, emm12 442 and emm89 common among UK scarlet fever cases and upper respiratory tract infections (35,

443 38). Very similar patterns of emm-types causing invasive disease are also found in other 444 European countries and North America, with emm1, emm28, emm89, emm3, emm12, emm4 and emm6 leading (39). The genotype emm 108 has not previously been reported to be a common emm-type in the UK or elsewhere, but reported in $2018 / 2019$ by Public Health

447 England to be a cause of national upsurges in infections in England/Wales

448 (https://assets.publishing.service.gov.uk/government/uploads/system/uploads/attachment_dat 449 a/file/800932/hpr1619_gas-sf3.pdf). The data on prevalence of this emm-type is based on invasive disease data, as only invasive infections are notifiable in England/Wales. From the available data it is not clear if it would have been common among throat infections during this

452 time as well as skin infections, but suggests it is not unique to our sampled geographical region 453 of Sheffield, UK.

454 The emm-pattern D, previously determined to be associated with skin infections, was the most 
457 found that among impetigo isolates, $49.8 \%$ were $\mathrm{D}, 42 \%$ were $\mathrm{E}$ and $8.2 \%$ were $\mathrm{A}-\mathrm{C}$ patterns, we found a higher level of A-C isolates (29\%) in HIC isolates. This could be due to the more diverse collection of HIC isolates, given that we did not focus specifically on impetigo. Interestingly, the dominant $\mathrm{HIC}$ emm-types were either pharyngeal specialist pattern A-C (emm 1 and emm12) or generalist pattern $\mathrm{E}(\mathrm{emm} 4$ and emm89), with only emm 108 representing skin specialist pattern D.

In the LIC isolates, all six E emm-clusters were represented, with the most common being E6 (18\%) closely followed by E4 (16\%) and E3 (14\%). E6 was recently found to be the leading cluster in Gambian non-invasive isolates (skin and pharyngeal) but with E3 leading among invasive isolates (9). D4 was also common in LIC isolates (17\%) but, more so in HIC isolates where $35 \%$ of the isolates were D4. This was almost equal to all E clusters combined, but again explained by the high number of emm 108 isolates. A higher number of singleton emm-cluster 471 types were also found in the LIC isolates $(\mathrm{n}=17)$ representing $9 \mathrm{emm}$-types, compared to HIC 472 isolates $(\mathrm{n}=11)$ representing just two emm-types. There was an association with E emm-cluster 473 isolates also carrying the sof gene, as all E1-E4 emm-types were sof positive. Four LIC E6 emm-types (emm46, 65, 182 and 205) were sof negative and all E5 emm-types were negative. 475 HIC emm12 isolates carried a sof gene that would only produce a truncated form of SOF, as 476 previously identified (11).

477 Consistent with the high number of D/E pattern isolates, we also found the majority of isolates

478 had the Mga-regulon pattern I, and therefore carried the emm-like genes mrp and enn. Within the HIC emm4 isolates we found that 4/9 carried the emm-enn fusion gene, and this was also 
associated with degraded prophages in these isolates $(40,41)$. Given the high number of isolates carrying Mrp and Enn it is possible that they contribute to pathogenesis at the same, or even greater, level of the M protein (28). The M-like proteins have not been well characterised and their role and expression may vary depending on the allele or other genetic factors. The existence of two major clades within the Mrp and Enn phylogeny is of interest and may indicate varying domains and functions. Despite being adjacent to the emm gene, we did not observe sharing of enn and mrp alleles with emm-type over the two geographical sites. We did, however, see the same allele or very closely related alleles of mrp and enn shared with different emm-types across different geographical locations.

HIC emm4 and emm89 isolates were acapsular, as expected, but this was not the case for LIC emm4 and emm89, again reflecting very different genetic backgrounds. All LIC isolates carried the has $A B C$ genes required to synthesise the capsule, only one isolate had a mutation that would lead to a truncated HasA and a probably acapsular phenotype.

The FCT region encodes for genes thought to be involved in adhesion to the host, particularly for pili construction are encoded within the FCT and include a major pilus subunit, one or two minor subunits, at least one specific sortase and a chaperone (42). The pili of the M1 isolate,

497 SF370, has been shown to be essential for adherence to human tonsil and human skin (43), indicating its role in primary interactions and establishing infection. Other factors included within the FCT region are fibrinogen and fibronectin binding proteins, which may also contribute to host cell interactions, as well as transcriptional regulators. We identified the

501 previously described FCT types FCT1-6 and FCT9 among our isolates but, also a new FCT 502 type (FCT10) that was based on FCT5 with an additional fibronectin binding protein. FCT2 and FCT6 was restricted to HIC isolates and the new FCT10 was only found in LIC isolates. 
504 FCT3 and FCT4 were the most common types across both sites, found in 70\% (16/23) and 74\%

505 (34/46) of emm-types, representing 54\% (76/142) and 69\% (74/107) HIC and LIC isolates,

506 respectively. FCT3 and FCT4 have been shown to share the greatest similarity and can undergo

507 recombination (42). Both these FCTs have a cpa gene, which encodes for a collagen binding

508 subunit found at the pilus tip, one or two fibronectin-binding proteins $(s f b I / s f b I I)$ and the

509 regulator $m s m R$ upstream of the fibronectin-binding protein. The pilus and fibronectin-binding

510 proteins may contribute to tissue-specific host cell adhesion, in addition to others located

511 outside the FCT region. This includes $f b a A$, which we identified to present in all isolates except

512 for the majority of A-C pattern types, and has been found to contribute to skin infection (31).

513 The regulator $m s m R$ has been shown to have a positive effect on the fibronectin binding protein

514 expression and may also control other surface proteins, impacting on host cell adhesion (44).

515 It is not clear if specific FCT types confer tissue tropism and previous work has shown that

516 there is a high level of variability in host cell interactions and biofilm formation between

517 isolates sharing the same FCT (45). This indicates that there are other bacterial factors involved

518 in the expression of FCT related genes. The role of the regulators $n r a$ or rofA do vary between

519 isolates of differing genetic backgrounds, with evidence of environmental effects such as $\mathrm{pH}$

520 and temperature (42). We explored the sequences of rofA, $n r a$ and $m s m R$ and found a number

521 of different variations, however, many seemed to be related to emm-type and it is difficult to

522 determine if any variation would impact on function. This was also the case for the two-

523 component regulator CovR/S and the regulator of $c o v$, RocA, for which variations can impact

524 on the expression of a number of virulence factors. Variations in CovS and RocA were common

525 among both LIC and HIC isolates but the transcriptional impact of any of these amino acid

526 changes is unclear. Only one HIC isolate had an amino acid difference in CovR (M17I, emm77)

527 and one other HIC isolate had a premature stop codon in CovS; both may alter expression of

528 virulence genes. Whether there are differences in expression and control of FCT and other 
529 virulence factor genes in LIC isolates compared to HIC isolates and/or between skin infection

530 isolates and other types of infection isolates is yet to be determined. Inclusion of isolates

531 causing other infections, such as pharyngeal infection isolates may reveal some tissue tropism

532 differences or factors. However, the complex nature of regulatory systems also makes it

533 difficult to determine the impact of single amino acid variants and control of transcription may

534 vary between emm-types.

535 Superantigens are important $S$. pyogenes virulence factors and their distribution may differ

536 between isolates. The chromosomal speG and smeZ genes were the most common in both

537 populations, with more than $90 \%$ of the isolates carrying these genes. The prophage-associated

538 speC and ssa were more common in HIC isolates compared to LIC isolates, and three HIC

539 isolates actually carried two copies of $s p e C$, along with the DNase $s p d 1$, on two separate

540 prophages. Typically, speA is prophage associated but the divergent speA.4 allele is associated

541 with a prophage-like element that has been previously only found in in emm6, emm32, emm67

542 and emm77 (32). We found this only in the HIC emm6, but, although speA was almost equally

543 as common in the LIC population, all, except one, of the 22 speA-positive LIC isolates carried

544 speA.4 associated with the prophage-like element. Only a LIC emm89 isolate carried speA on

545 what appeared to be a complete prophage and was only one base pair different from the speA.1

546 allele. Interestingly, we also identified a gene in one LIC isolate (emm65) that appeared to be

547 a fusion of 5' speK and 3' speM, and since speK and speM are phage encoded, it could be a

548 result of recombination of phages carrying the two genes. BLASTp of this potential fusion

549 protein identified a similar (two-three amino acid different) variant in six published genomes;

550 NS88.3 (emm98, locus accession PWO34032), emm89.14 (QCK42181), emm100

551 (QCK70992), NS426 (VGQ95836), NS76 (VGR28970) and NS6221 (VHG25078). 
552

553

554

555

556

557

558

559

560

561

562

563

564

565

566

567

568

569

570

571

572

573

574

575

576

Only two of the prophage-associated DNases ( $s p d 1$ and $s p d 3$ ) were found in the LIC isolates, while five DNases (sdal, sda2, sdn, spdl, spd3 and spd4) were identified in the HIC isolates. Almost all $(136 / 142,96 \%)$ of the HIC population carried at least one prophage-associated DNase, whereas only two LIC isolates carried $s p d 3$ and only $24 \%$ of isolates carried $s p d 1$, which associated with the superantigen $s p e C$. DNases, such as sdal, have been shown to be necessary and sufficient to degrade neutrophil extracellular traps (46), therefore the lack of these in LIC isolates from The Gambia could be suggestive of limited/reduced ability of immune evasion, and warrants further investigation into their invasive capacity. There is the potential that other prophage-associated DNases exist but are yet to be identified. It also suggests differences in circulating phages between the two sites, although the accessory genome appeared to be much greater in LIC isolates compared to HIC isolates. This could be related to the high prevalence of tetracycline resistance genes within the LIC population that may be carried on mobile genetic elements. Further investigation is needed to determine prophage content, as well as other mobile genetic elements; this is, however, notoriously difficult with short read sequence data and may require supporting long read data.

The most advanced multi-valent $S$. pyogenes experimental vaccine is based on $30 \mathrm{emm}$-types identified from isolates causing infection predominantly in high income countries $(4,5)$. Based on the emm-types distributions, we determine the direct coverage of the vaccine to be only $24 \%$ in the LIC population, compared to $61 \%$ in the HIC population, although we did not explore cross-reactivity between emm-types. The high proportion of emm108 in HIC isolates was unexpected as this was not a previously recognised dominant emm-type and highlights the potential for sudden and dramatic increases in new emm-types that could escape a serotypespecific vaccine. If such a vaccine was introduced, monitoring of new variants in the noninvasive as well as the invasive bacterial populations would be needed, and on a global scale. Alternatively, a vaccine targeting antigens with limited variability between isolates may be 
577 preferable, if these can still provide similar levels of protection. We have confirmed that several 578 previously identified potential targets (37) are also highly conserved in our LIC and HIC 579 bacterial populations. However, both our LIC and HIC isolates represent only single 580 geographical locations: Sukuta, The Gambia and Sheffield, UK. Further in-depth genomic 581 analysis of international S. pyogenes populations, encompassing more LICs and different infection types, is needed to confirm diversity and distribution of potential vaccine diversity.

583 Our study confirms work by others (37), that emm-typing alone is insufficient to comprehensively characterise global isolates. Furthermore, genetic features that have been characterised in particular HIC emm-types, such as the absence of the has $A B C$ locus in emm4, may not be present in LIC isolates of the same genotype. In the absence of WGS, other

587 molecular markers, such as MLST, enn, mrp and FCT type could be used in addition to emmtyping to characterise the diverse genetic background of isolates from different geographical settings. More work is required to understand why there is such a high genetic diversity in LIC settings compared to HIC and with limited overlap. This may be linked to infection types but

591 there is insufficient data both on pharyngeal infections in LICs, like The Gambia, as well as

592 skin infections in HICs. By increasing the characterisation of isolates from different infections

593 over wider geographical settings we could gain real insight into the molecular mechanisms underpinning tissue tropism.

\section{Contributors}

596 E.P.A, M.M and T.I.d.S coordinated collection of the Gambian isolates; L.T coordinated 597 collection of the UK isolates; S.Y.B, A.J.K, E.S, S.D, L.T and H.K cultured bacterial isolates 598 and extracted genomic DNA; J.M and A.K.S performed the whole genome sequencing of the

599 Gambian isolates; S.Y.B and C.E.T performed the whole genome sequencing analyses with 
assistance from R.R.C.; S.Y.B, C.E.T and T.I.d.S secured funding for the project; S.Y.B and

\section{Competing interests}

603 The authors declare that there are no competing interests.

\section{Acknowledgements}

605 We would like to thank the invaluable contribution of the members of the MRCG Strep A 606 Study Group whose names are not in the main authorship list: Annette Erhart; Pierre R 607 Smeesters; Martin Antonio; Sona Jabang; Beate Kampmann; Anna Roca; Isatou Jagne Cox; Peggy-Estelle Tiencheu; Grant Mackenzie.

609 This work is supported by Global Challenge Research Fund obtained through the University 610 of Sheffield (S.Y.B). The authors also thank the MRCG at LSHTM and study participants.

611 C.E.T is a Royal Society \& Wellcome Trust Sir Henry Dale Fellow (208765/Z/17/Z). T.I.d.S is supported by a Wellcome Trust Intermediate Clinical Fellowship (110058/Z/15/Z). E.P.A is supported by a Wellcome Trust Clinical PhD fellowship in Global Health (222927/Z/21/Z).

614 The authors thank Prof Pierre Smeesters and Dr Anne Botteaux (Universite Libre de Bruxelles)

615 for kindly providing us with new allele numbers for enn and mrp.

\section{References:}

617 1. Walker MJ, Barnett TC, McArthur JD, Cole JN, Gillen CM, Henningham A, Sriprakash KS, Sanderson-Smith ML, Nizet V. (2014). Disease manifestations and pathogenic mechanisms of group A Streptococcus. Clin Microbiol Rev 27:264-301. 
disease, 1990-2015. N Engl J Med. 377:713-722.

625

3. Vekemans J, Gouvea-Reis F, Kim JH, Excler JL, Smeesters PR, O’brien KL, Van 883.

4. Dale JB, Penfound TA, Chiang EY, Walton WJ. (2011) New 30-valent M proteinbased vaccine evokes cross-opsonic antibodies against non-vaccine serotypes of group A streptococci. Vaccine 29. 46:8175-8178.

5. Pastural É, McNeil SA, MacKinnon-Cameron D, Ye L, Langley JM, Stewart R, Martin LH, Hurley GJ, Salehi S, Penfound TA, Halperin S, Dale JB. (2020) Safety and immunogenicity of a 30-valent $\mathrm{M}$ protein-based group a streptococcal vaccine in healthy adult volunteers: A randomized, controlled phase I study. Vaccine 38. 6:1384192.

6. Steer AC, Law I, Matatolu L, Beall BW, Carapetis JR. (2009) Global emm type distribution of group A streptococci: systematic review and implications for vaccine development. Lancet Infect Dis. 9:611-616.

641 7. Seale AC, Davies MR, Anampiu K, Morpeth SC, Nyongesa S, Mwarumba S, Dougan G, Berkley JA. (2016) Invasive group A Streptococcus infection among children, rural Kenya. Emerg Infect Dis 22:224-232. Africa to inform vaccine development. mSphere 5:e00429-20.

648 9. Jabang S, Erhart A, Darboe S, Baldeh AK, Delforge V, Watson G, Foster-Nyarko E, 
10. Bessen DE, Smeesters PR, Beall BW. (2018) Molecular Epidemiology, Ecology, and Evolution of Group A Streptococci. Microbiol Spectr 6.

11. Bessen DE, McShan WM, Nguyen SV, Shetty A, Agrawal S, Tettelin H. (2015)

12. Bessen DE, Carapetis JR, Beall B, Katz R, Hibble M, Currie BJ, Collingridge T, Izzo MW, Scaramuzzino DA, Sriprakash KS. (2000) Contrasting molecular epidemiology of group A streptococci causing tropical and nontropical infections of the skin and throat. J Infect Dis 182:1109-16.

13. Carapetis JR, Steer AC, Mulholland EK, Weber M. (2005) The global burden of group A streptococcal diseases. Lancet Infect Dis. 5:685-94 variation of paediatric scabies and pyoderma prevalence in the Gambia: A crosssectional study. PLoS Negl Trop Dis. 13:e0007801. positive bacteria. Trends Genet. 11:217-8.

673 17. Bolger AM, Lohse M, Usadel B. (2014) Trimmomatic: A flexible trimmer for Illumina 
sequence data. Bioinformatics 30:2114-20.

675

676

677

678

679

680

681

682

683

684

685

686

687

688

689

690

691

692

693

694

695

696

697

698

18. Bankevich A, Nurk S, Antipov D, Gurevich AA, Dvorkin M, Kulikov AS, Lesin VM, Nikolenko SI, Pham S, Prjibelski AD, Pyshkin A V., Sirotkin A V., Vyahhi N, Tesler G, Alekseyev MA, Pevzner PA. (2012) SPAdes: A new genome assembly algorithm and its applications to single-cell sequencing. J Comput Biol. 19:455-477.

19. Gurevich A, Saveliev V, Vyahhi N, Tesler G. (2013) QUAST: Quality assessment tool for genome assemblies. Bioinformatics. 29:1072-5.

20. Seemann T. (2014) Prokka: Rapid prokaryotic genome annotation. Bioinformatics. 30:2068-9.

21. Page AJ, Cummins CA, Hunt M, Wong VK, Reuter S, Holden MTG, Fookes M, Falush D, Keane JA, Parkhill J. (2015) Roary: Rapid large-scale prokaryote pan genome analysis. Bioinformatics. 31:3691-3.

22. Stamatakis A. (2014) RAxML version 8: a tool for phylogenetic analysis and postanalysis of large phylogenies. Bioinformatics. 30:1312-1313.

23. Letunic I, Bork P. (2019) Interactive Tree of Life (iTOL) v4: Recent updates and new developments. Nucleic Acids Res. 47:W256-W259.

24. Li H, Durbin R. (2009) Fast and accurate short read alignment with Burrows-Wheeler transform. Bioinformatics. 25:1754-60.

25. Gupta SK, Padmanabhan BR, Diene SM, Lopez-Rojas R, Kempf M, Landraud L, Rolain JM. (2014) ARG-ANNOT, a new bioinformatic tool to discover antibiotic resistance genes in bacterial genomes. Antimicrob Agents Chemother. 58:212-20.

26. Quinlan AR, Hall IM. (2010) BEDTools: A flexible suite of utilities for comparing genomic features. Bioinformatics. 26:841-2.

27. Assefa S, Keane TM, Otto TD, Newbold C, Berriman M. (2009) ABACAS: algorithmbased automatic contiguation of assembled sequences. Bioinformatics. 25:1968-9. 
699

700

701

702

703

704

705

706

707

708

709

710

711

712

713

714

715

716

717

718

719

720

721

722

723

28. Frost HR, Davies MR, Delforge V, Lakhloufi D, Sanderson-Smith M, Srinivasan V, Steer AC, Walker MJ, Beall B, Botteaux A, Smeesters PR. (2020) Analysis of Global Collection of Group A Streptococcus Genomes Reveals that the Majority Encode a Trio of M and M-Like Proteins. mSphere. 5:e00806-19.

29. Falugi F, Zingaretti C, Pinto V, Mariani M, Amodeo L, Manetti AGO, Capo S, Musser JM, Orefici G, Margarit I, Telford JL, Grandi G, Mora M. (2008) Sequence Variation in Group A Streptococcus Pili and Association of Pilus Backbone Types with Lancefield T Serotypes . J Infect Dis. 198:1834-41.

30. Sanderson-Smith M, De Oliveira DMP, Guglielmini J, McMillan DJ, Vu T, Holien JK, Henningham A, Steer AC, Bessen DE, Dale JB, Curtis N, Beall BW, Walker MJ, Parker MW, Carapetis JR, Van Melderen L, Sriprakash KS, Smeesters PR, Batzloff M, Towers R, Goossens H, Malhotra-Kumar S, Guilherme L, Torres R, Low D, McGeer A, Krizova P, El Tayeb S, Kado J, Van Der Linden M, Erdem G, Moses A, Nir-Paz R, Ikebe T, Watanabe H, Sow S, Tamboura B, Kittang B, Melo-Cristino J, Ramirez M, Straut M, Suvorov A, Totolian A, Engel M, Mayosi B, Whitelaw A, Darenberg J, Normark BH, Ni CC, Wu JJ, De Zoysa A, Efstratiou A, Shulman S, Tanz R. (2014) A systematic and functional classification of Streptococcus pyogenes that serves as a new tool for molecular typing and vaccine development. J Infect Dis. 210:1325-38.

31. Rouchon CN, Ly AT, Noto JP, Luo F, Lizano S, Bessen DE. (2017) Incremental Contributions of FbaA and Other Impetigo-Associated Surface Proteins to Fitness and Virulence of a Classical Group A Streptococcal Skin Strain. Infect Immun. 85:e0037417.

32. Banks DJ, Porcella SF, Barbian KD, Beres SB, Philips LE, Voyich JM, DeLeo FR, Martin JM, Somerville GA, Musser JM. (2004) Progress toward characterization of the group A Streptococcus metagenome: Complete genome sequence of a macrolide- 
resistant serotype M6 strain. J Infect Dis. 190:727-38.

725

726

727

728

729

730

731

732

33. Turner CE, Holden MTG, Blane B, Horner C, Peacock SJ, Sriskandan S. (2019) The emergence of successful Streptococcus pyogenes lineages through convergent pathways of capsule loss and recombination directing high toxin expression. MBio. 10:e02521-19.

34. Kratovac Z, Manoharan A, Luo F, Lizano S, Bessen DE. (2007) Population genetics and linkage analysis of loci within the FCT region of Streptococcus pyogenes. J Bacteriol. 189:1299-310.

35. Lynskey NN, Jauneikaite E, Li HK, Zhi X, Turner CE, Mosavie M, Pearson M, Asai M, Lobkowicz L, Chow JY, Parkhill J, Lamagni T, Chalker VJ, Sriskandan S. (2019) Emergence of dominant toxigenic M1T1 Streptococcus pyogenes clone during increased scarlet fever activity in England: a population-based molecular epidemiological study. Lancet Infect Dis. 19:1209-1218.

36. Frost HR, Laho D, Sanderson-Smith ML, Licciardi P, Donath S, Curtis N, Kado J, Dale JB, Steer AC, Smeesters PR. 2017. Immune Cross-Opsonization within emm clusters following group A Streptococcus skin infection: Broadening the scope of typespecific immunity. Clin Infect Dis. 65:1523-1531.

37. Davies MR, McIntyre L, Mutreja A, Lacey JA, Lees JA, Towers RJ, Duchêne S, Smeesters PR, Frost HR, Price DJ, Holden MTG, David S, Giffard PM, Worthing KA, Seale AC, Berkley JA, Harris SR, Rivera-Hernandez T, Berking O, Cork AJ, Torres RSLA, Lithgow T, Strugnell RA, Bergmann R, Nitsche-Schmitz P, Chhatwal GS, Bentley SD, Fraser JD, Moreland NJ, Carapetis JR, Steer AC, Parkhill J, Saul A, Williamson DA, Currie BJ, Tong SYC, Dougan G, Walker MJ. (2019) Atlas of group A streptococcal vaccine candidates compiled using large-scale comparative genomics. Nat Genet. 51:1035-1043. 
38. Chalker V, Jironkin A, Coelho J, Al-Shahib A, Platt S, Kapatai G, Daniel R, Dhami C, Laranjeira M, Chambers T, Guy R, Lamagni T, Harrison T, Chand M, Johnson AP, Underwood A, Ramsay M, Fry N, Purohit A, Brown R. (2017) Genome analysis following a national increase in Scarlet Fever in England 2014. BMC Genomics. $18: 224$

39. Gherardi G, Vitali LA, Creti R. (2018) Prevalent emm-types among invasive GAS in Europe and North America since year 2000. Front Public Health. 6:59.

40. DebRoy S, Li X, Kalia A, Galloway-Pena J, Shah BJ, Fowler VG, Flores AR, Shelburne SA. (2018) Identification of a chimeric emm gene and novel emm pattern in currently circulating strains of emm4 Group A Streptococcus. Microb Genom. 4:e000235.

41. Remmington A, Haywood S, Edgar J, Green LR, de Silva T, Turner CE. (2021) Cryptic prophages within a Streptococcus pyogenes genotype emm4 lineage. Microb Genom. 7:mgen000482.

42. Nakata M, Kreikemeyer B. (2021) Genetics, Structure, and Function of Group A Streptococcal Pili. Front Microbiol. 12:616508.

43. Abbot EL, Smith WD, Siou GPS, Chiriboga C, Smith RJ, Wilson JA, Hirst BH, Kehoe MA. (2007) Pili mediate specific adhesion of Streptococcus pyogenes to human tonsil and skin. Cell Microbiol. 9:1822-33.

44. Nakata M, Podbielski A, Kreikemeyer B. (2005) MsmR, a specific positive regulator of the Streptococcus pyogenes FCT pathogenicity region and cytolysin-mediated translocation system genes. Mol Microbiol. 57:786-803.

45. Köller T, Manetti AGO, Kreikemeyer B, Lembke C, Margarit I, Grandi G, Podbielski A. (2010) Typing of the pilus-protein-encoding FCT region and biofilm formation as novel parameters in epidemiological investigations of Streptococcus pyogenes isolates 
from various infection sites. J Med Microbiol. 59:442-452.

775

46. Buchanan JT, Simpson AJ, Aziz RK, Liu GY, Kristian SA, Kotb M, Feramisco J, 

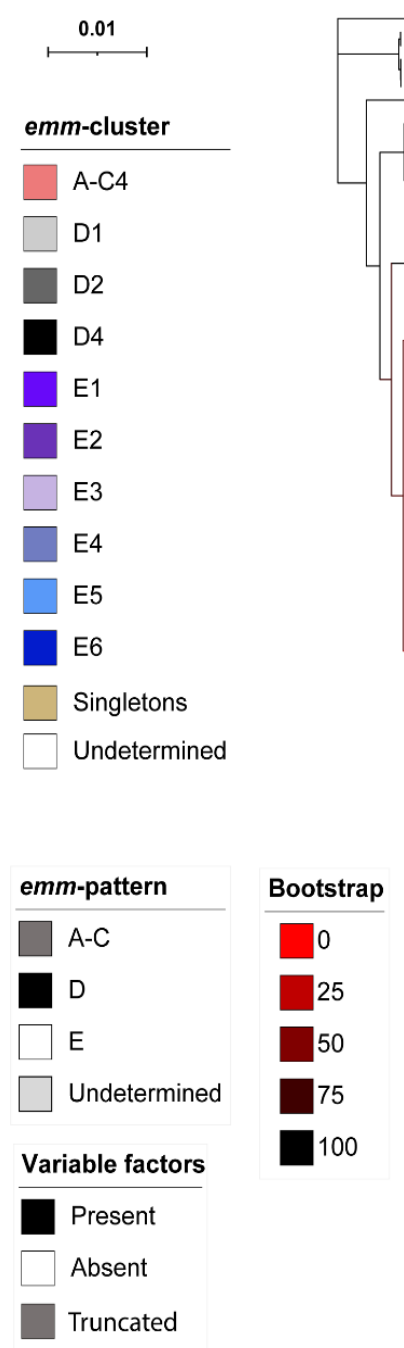
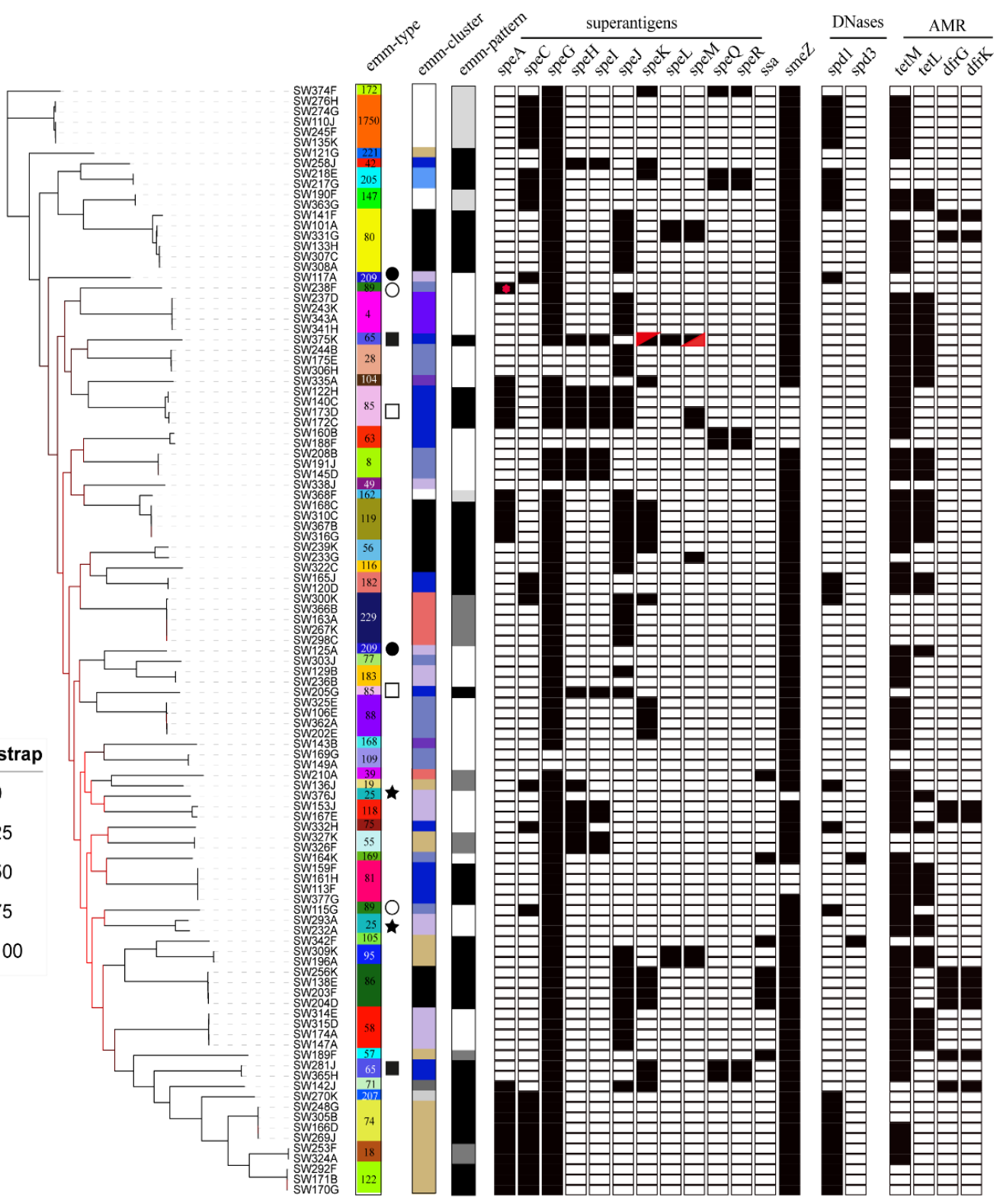

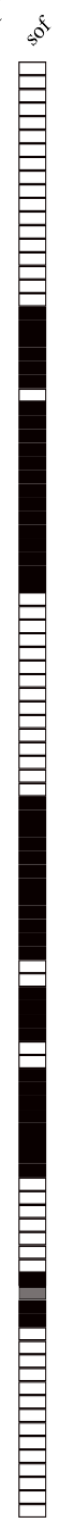

Figure 1: Phylogenetic analysis of 107 genomes from LIC isolates. A maximum likelihood

phylogeny was constructed from the core-gene alignment $(1,242,112 \mathrm{bp})$ using RAxML (22)

with 100 bootstraps. Isolates clustered by emm-type except those indicated, whereby two lineages were represented by a single emm genotype: star; emm 25 , filled square; emm65, open square; emm85, open circle; emm89, filled circle; emm209). Also shown is the presence (black)/absence (white) of the superantigen genes (speA, speC, speG, speH-M, speQ, speR, ssa and smeZ) and DNase genes spdl and spd3; four other DNase genes (sdal, sda2, sdn, and 
spd4) were tested for but were not found in any isolate. In all cases, except one (red dot), speA was located within the prophage-like element $\Phi 10394.2$. One isolate had a gene that appeared to be a fusion of 5' speK and 3' speM (red triangles). Antimicrobial resistance genes (AMR) tetM, tet $L, d f r G$ and $d f r K$ were also identified in some isolates (white; absent, black; present). The positivity for serum opacity factor ( $s o f$ ) is also shown, although for one emm55 isolate this gene would produce a truncated variant of SOF (grey). Scale bar represents substitutions per site. emm-types are coloured for easy visualisation and type numbers are also given. 


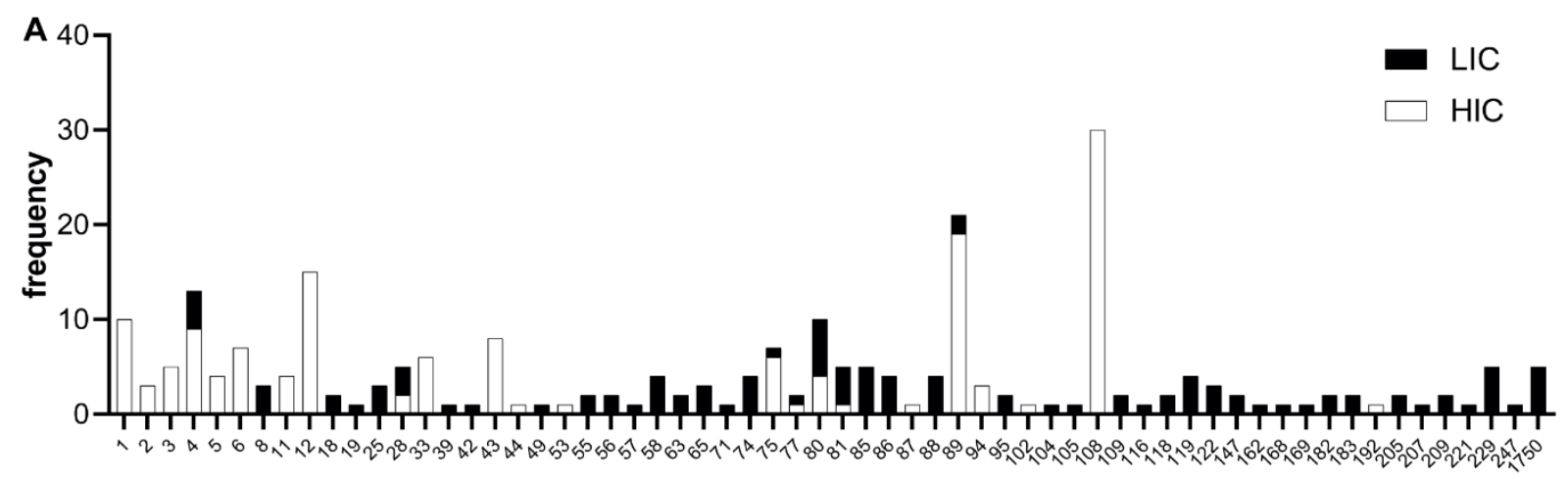

emm-type

B

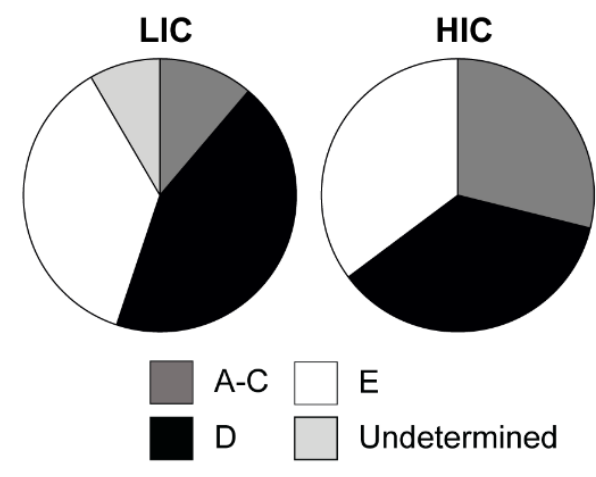

C
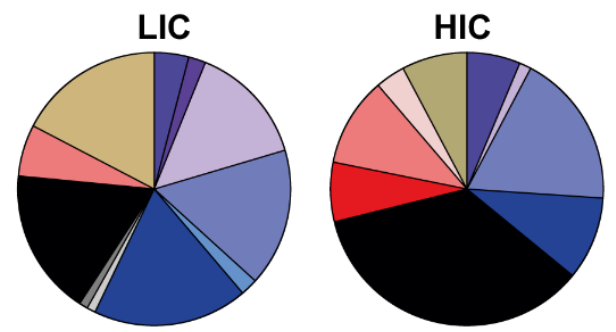

\begin{tabular}{|l|l|l|l|l|l}
\hline E1 & E4 $\square$ D1 & A-C3 $\square$ Singletons \\
E2 & $\square$ E5 & D2 & $\square$ A-C4 \\
E3 & E6 & D4 & $\square$ & A-C5
\end{tabular}

Figure 2. Distribution of emm-type, pattern and cluster differs by site. (A) The frequency of each of the $62 \mathrm{emm}$-types identified in the LIC isolates (Black) and the HIC isolates (White). (B) An emm-pattern of A-C, D or E was assigned to 98/107 LIC isolates (the remaining 9 were undetermined) and all $142 \mathrm{HIC}$ isolates. (C) An emm-cluster was also assigned to 98/107 LIC isolates (the remaining 9 were excluded) and all $142 \mathrm{HIC}$ isolates. Pie charts represent the percentage of isolates associated with each pattern/cluster. 

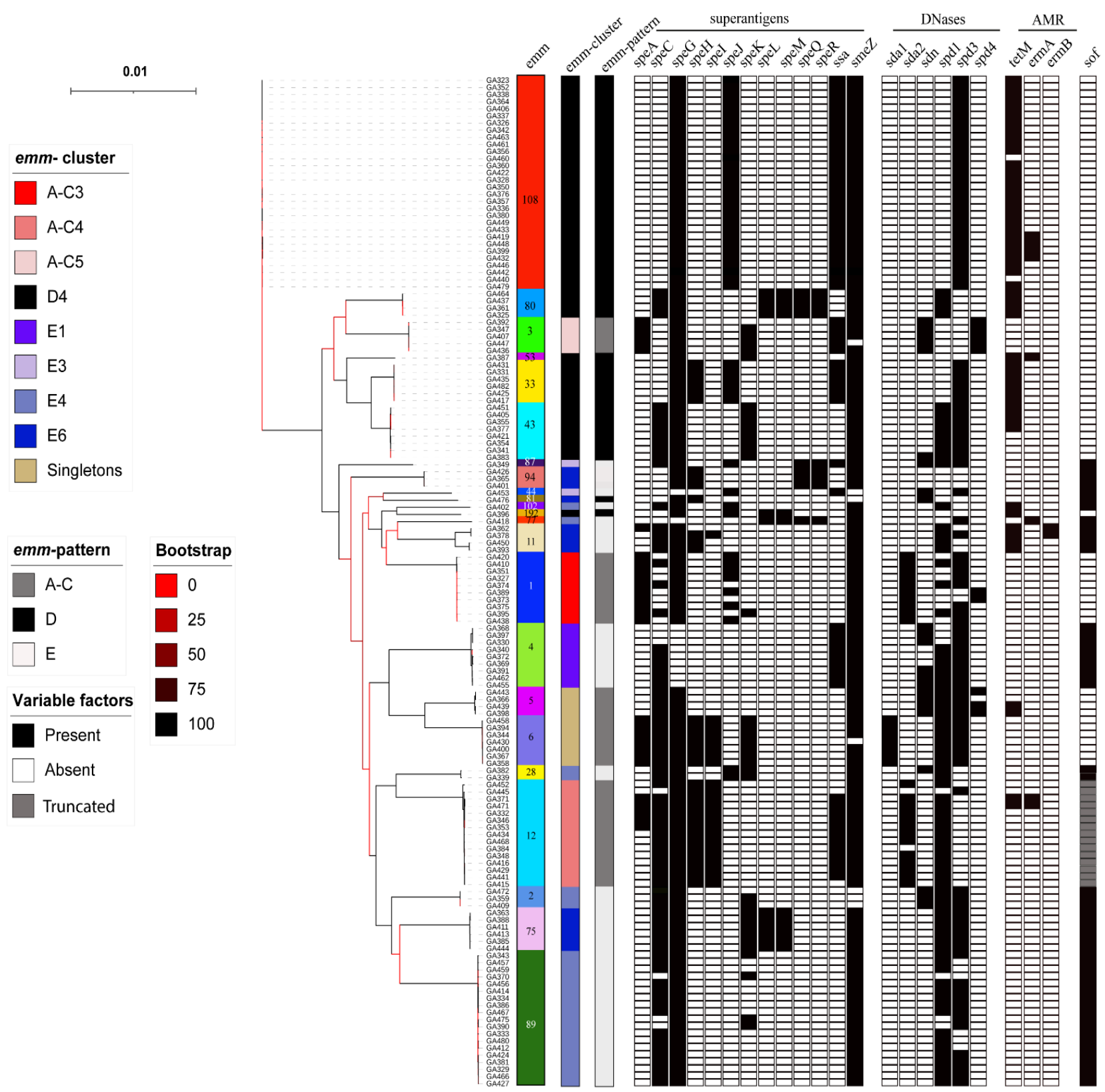

Figure 3: Phylogenetic analysis of 142 HIC isolates: A maximum likelihood phylogenetic

tree was generated with the core-gene alignment (1,202,105bp) using RAxML (22) with 100

bootstraps. All isolates clustered by emm-type. Presence (black)/absence (white) of

superantigens (speA, speC, speG, speH-M, speQ, speR, ssa and smeZ) and DNases (sdal,

sda2, sdn, spdl, spd3 and spd4) is indicated. Antimicrobial resistance genes (AMR) tetM,

erm $A$ and $\operatorname{erm} B$ were also identified in some isolates (white; absent, black; present). The

positivity for serum opacity factor (sof) is also shown, but in all emm 12 this gene would

produce a truncated variation of SOF (grey). Scale bar represents substitutions per site. emm- 
bioRxiv preprint doi: https://doi.org/10.1101/2021.09.10.459590; this version posted September 10, 2021. The copyright holder for this preprint (which was not certified by peer review) is the author/funder, who has granted bioRxiv a license to display the preprint in perpetuity. It is made available under aCC-BY 4.0 International license.

types are coloured for easy visualisation and type numbers are also given. 


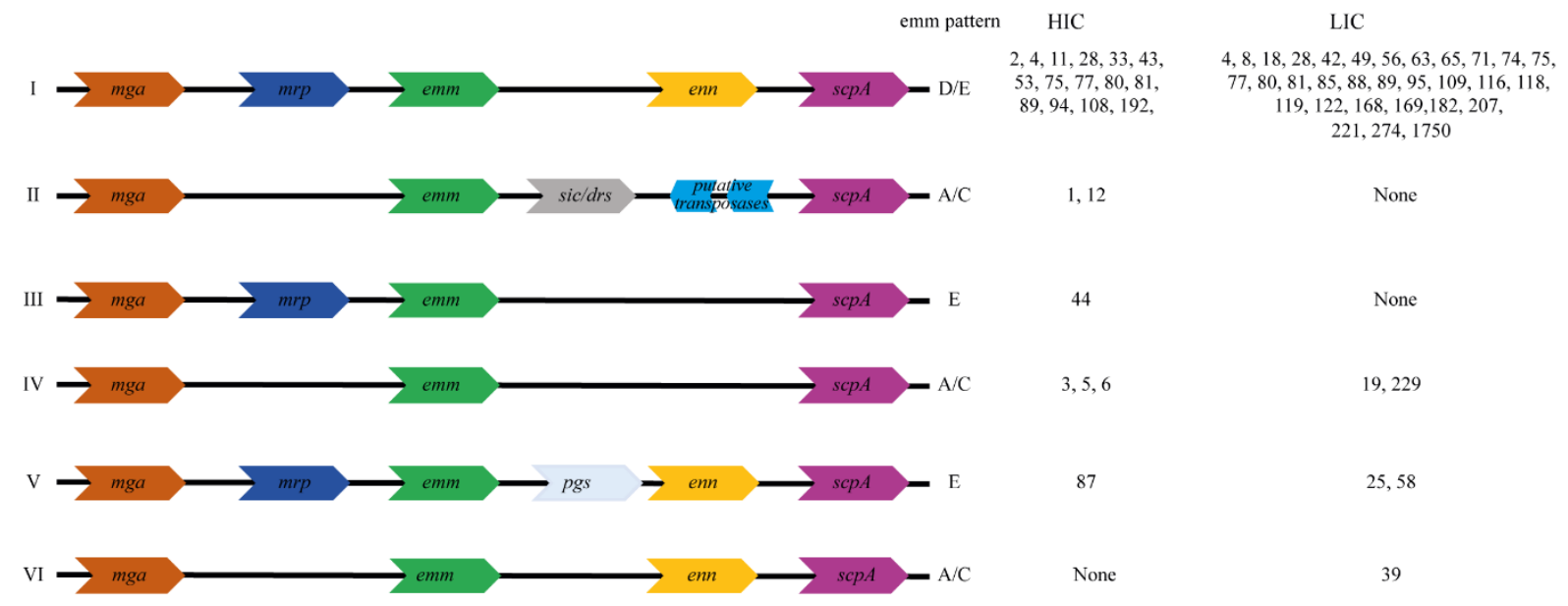

Figure 4: Arrangement of genes in the Mga regulon. The genes within the mga regulon for each isolate was determined and an Mga-regulon type I-VI assigned. The majority of emm types in both the HIC isolates and LIC isolates had type I with the M-like protein genes $m r p$ and enn flanking the M protein gene emm. The previously assigned emm pattern A-C/D/E (based on the emm type) is also given. The streptococcal inhibitor of complement (sic) gene was only identified in HIC emm 1 isolates, and the distantly related to sic (drs) gene found only in HIC emm 12 isolates. The gene pgs encodes for Pgs, a 15.5kDa protein of unknown function (28). 


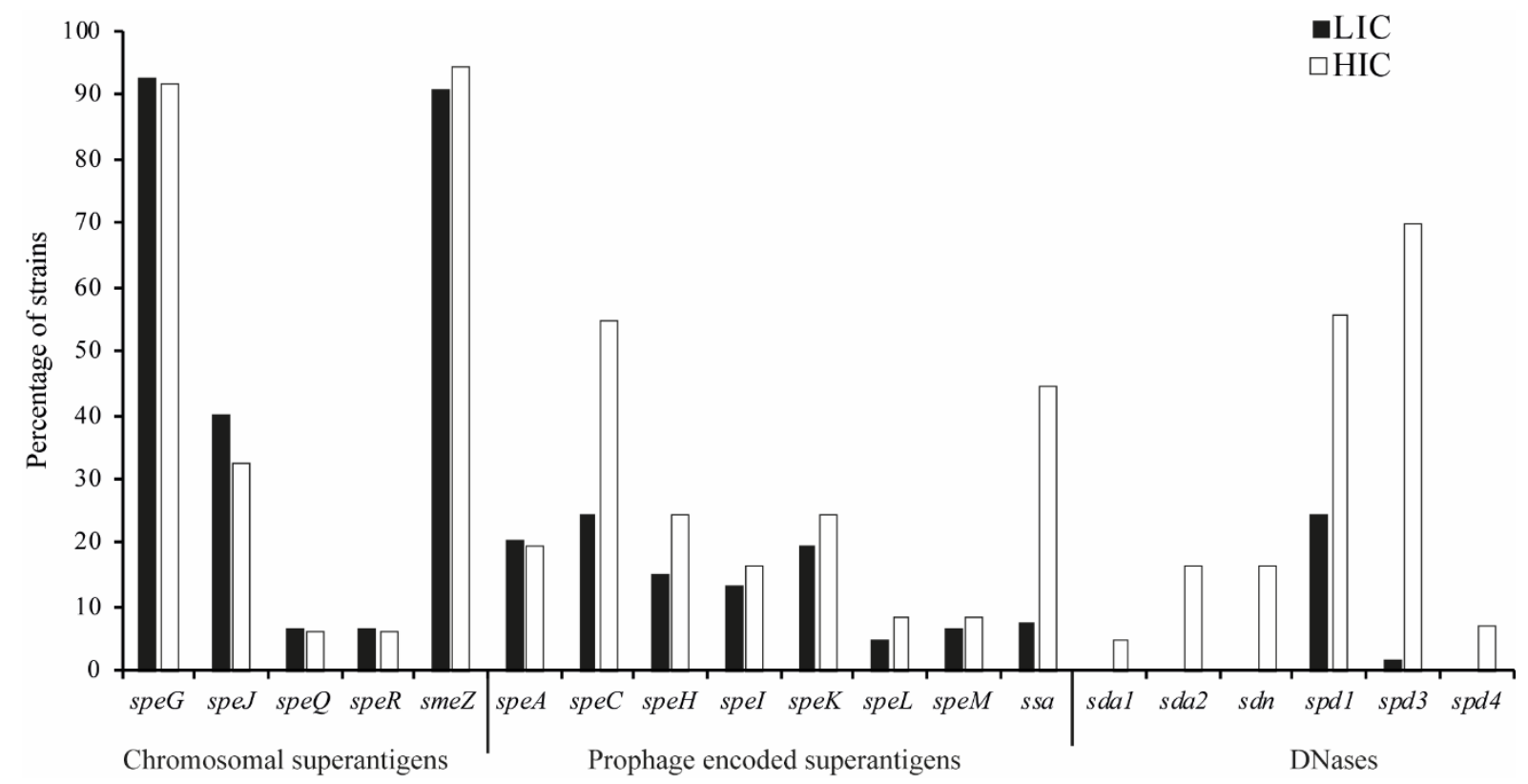

Figure 5: Superantigen and DNase gene carriage in LIC isolates compared to HIC

isolates. The proportions of the LIC isolates (black bars) and HIC isolates (white bars) carrying the respective genes determined by BLAST analysis and mapping. 


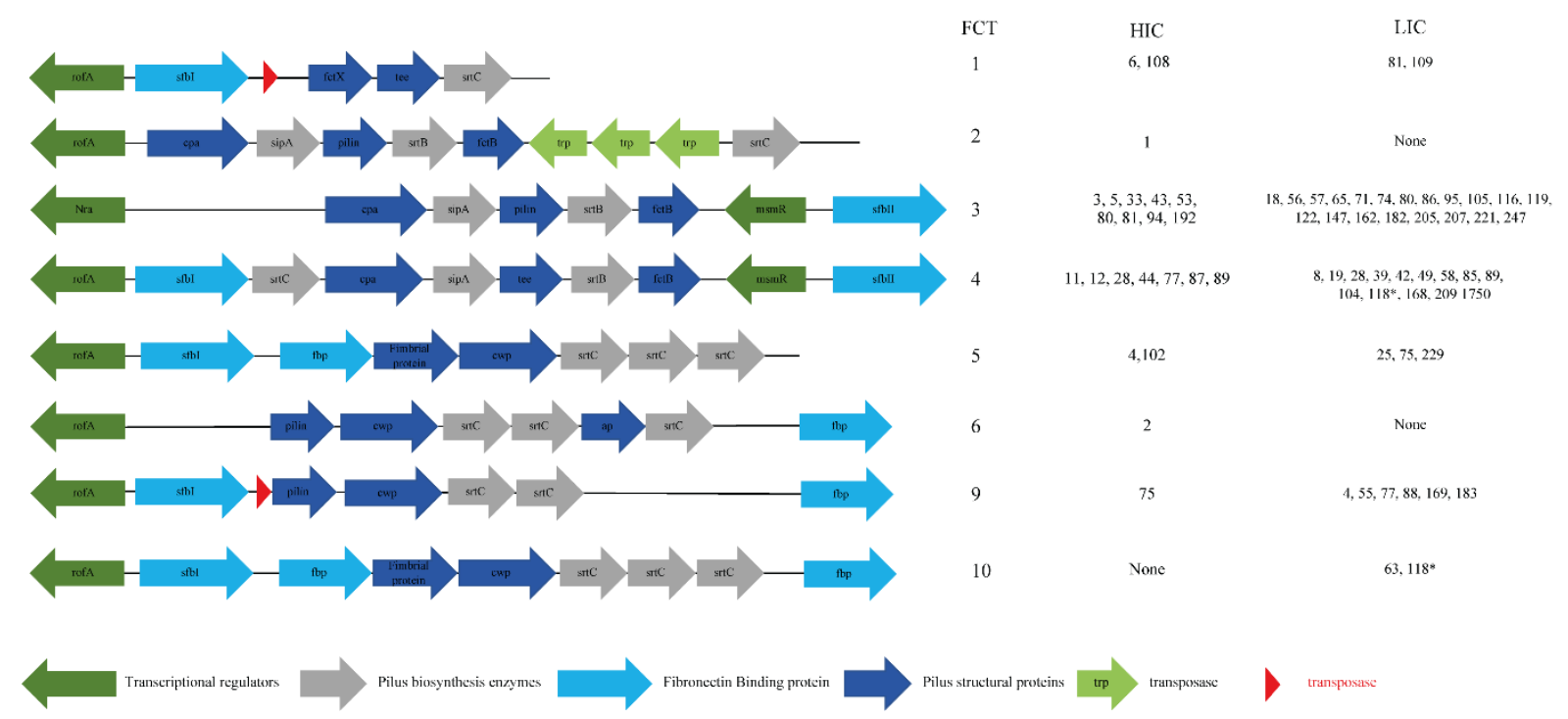

Figure 6: FCT arrangement patterns identified in LIC and HIC S. pyogenes isolates.

FCT regions were extracted from de novo assemblies and the FCT type assigned based on the predicted function and order of genes within the extracted region. The emm-types of isolates with each FCT type are shown for HIC and LIC isolates. A new FCT region was identified (FCT10) as similar to FCT5 but with an additional fibronectin binding protein after the sortase genes. For all emm-types there was at least one isolate with a designated FCT type in a single contiguous region. The only exception to this was emm118 $\left(^{*}\right)$ where the FCT was estimated to be FCT4 and the new FCT10 for each of the two isolates as the FCT region was split over two contigs. In FCT1 transposases were found in HIC emm6 and emm108, and in FCT9, transposases were found in HIC emm75 and LIC emm4. fbp; fibronectin binding protein, cwp; cell wall protein, ap; ancillary protein and trp; transposase. 


\section{Supplementary Figures}
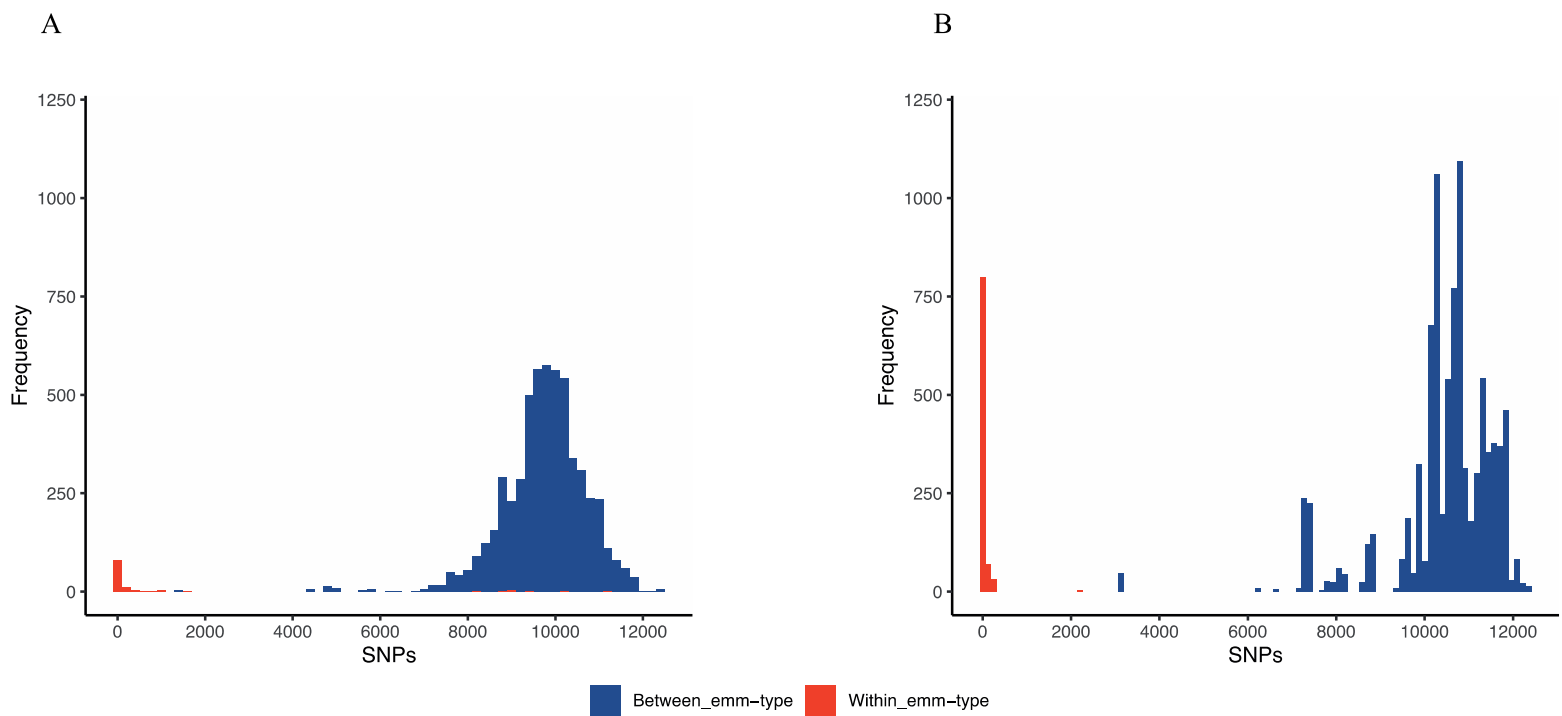

Supplementary Figure 1: Pairwise single nucleotide polymorphisms (SNPs) distances

SNPs were determined from the core-genome of (A) 107 LIC isolates and (B) 142 HIC isolates and pairwise distance calculated between isolates belonging to the same (red) or different (blue) emm-type. Overall, the median pairwise SNP distance within the same emm-type of LIC isolates was 22 (range $0-11,142$ SNPs), similar to that of HIC isolates with a median of 17 (range 0-2,206). Also comparable was the between emm-type median SNP distance; 9,816 (range 1,423-12,428) for LIC isolates, 11,110 (range 3,057-12,339) for LIC isolates. 


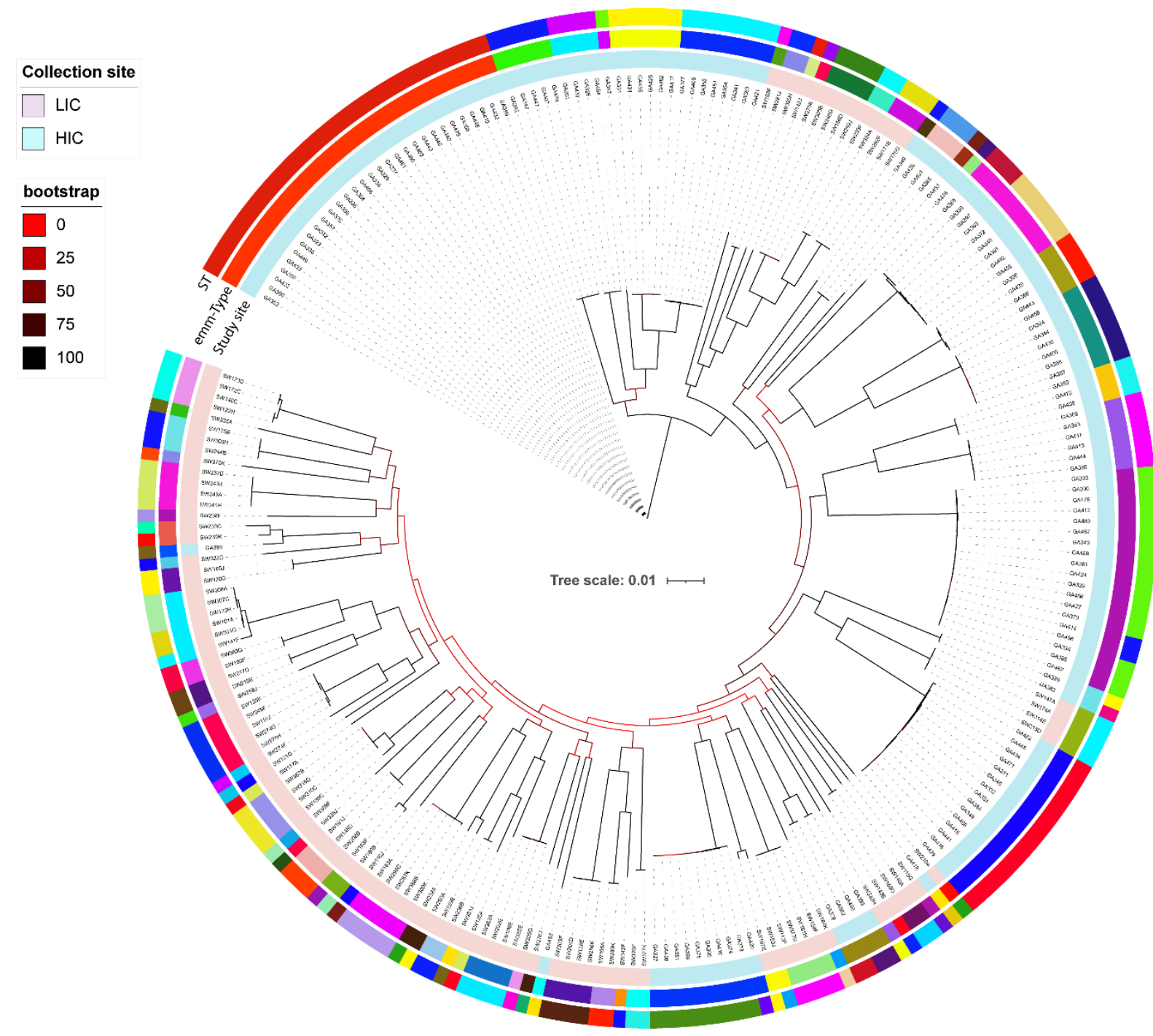

\section{Supplementary Figure 2: Population structure of the combined LIC (107) and HIC (142)}

isolates. A maximum likelihood phylogenetic was generated from the core-gene alignment (1,146,086bp) using RAxML with 100 bootstraps. Bootstrap support is indicated by colours in the legend. Inner circle: site of collection, middle circle: emm-types and outer circle: ST. 

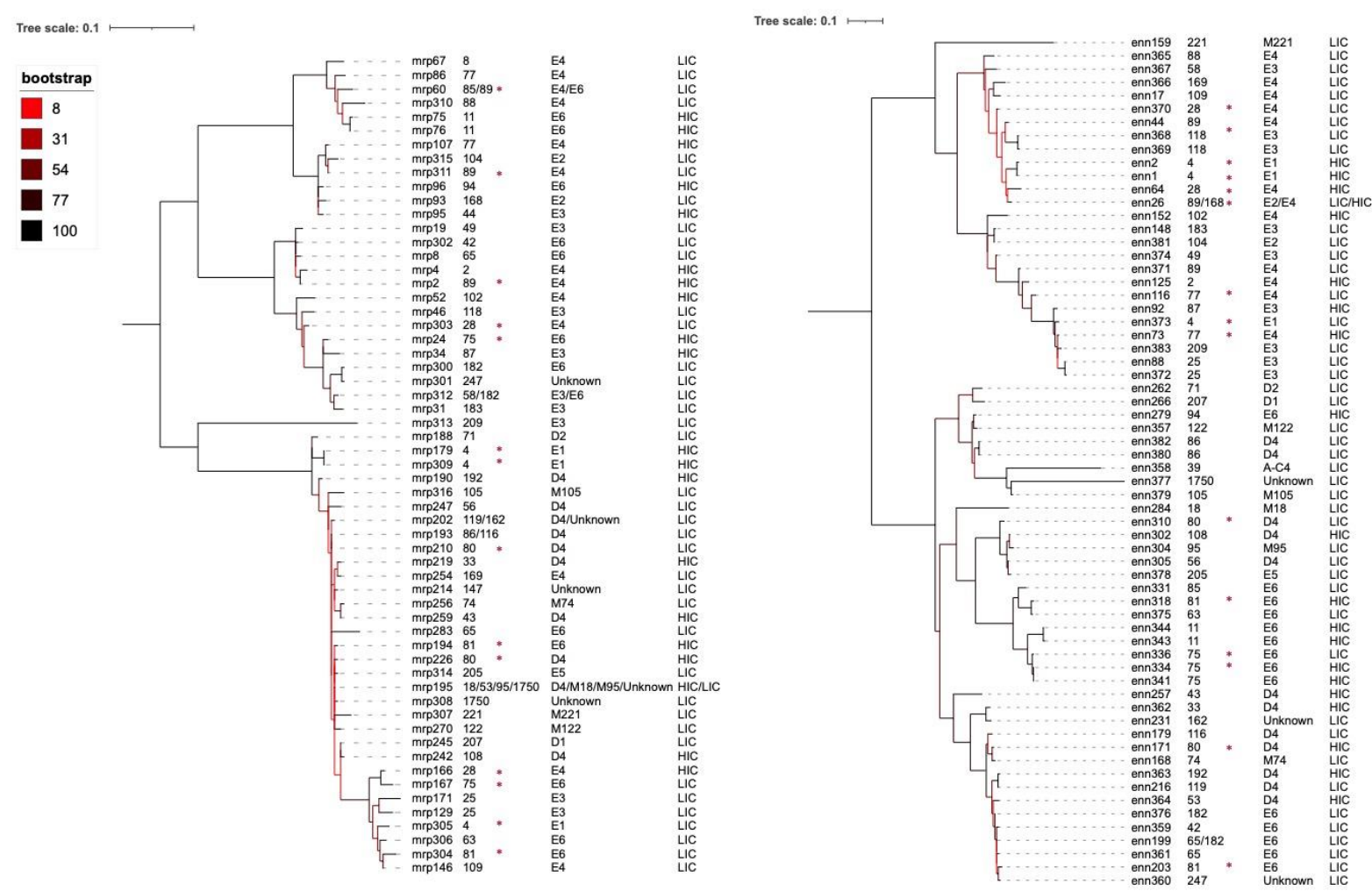

Supplementary Figure 3: Phylogenetic relatedness of unique Mrp (A) Enn (B) alleles. A maximum likelihood phylogenetic tree was generated from an amino acid alignment of unique Mrp or Enn alleles, using RAxML with 100 bootstraps (branch support shown by colour scale). The Mrp or Enn allele is shown followed by the associated emm-type(s), emm cluster(s) and population (LIC or HIC). * indicates the shared emm-types identified in both sites. 


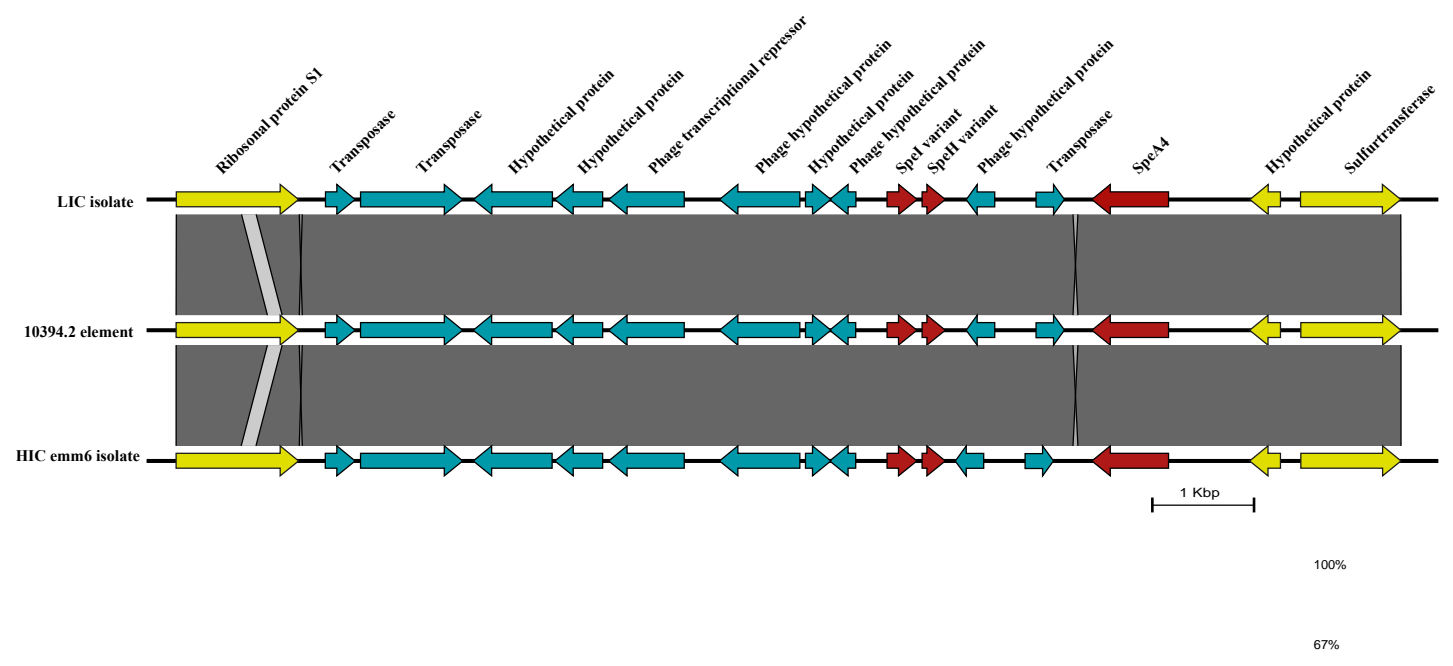

\section{Supplementary Figure 4: Comparison of 10394.2 phage-like element with the region}

found in HIC emm6 isolates and LIC isolates. The speA4 in LIC isolates and HIC emm6 were located within this phage-like element. This region also contains fragments of speI and speH. The same element was found in all LIC isolates that carried speA, except one that carried a different speA allele associated with a prophage. The corresponding regions were extracted from the respective isolate genomes and figure generated using EasyFig. 


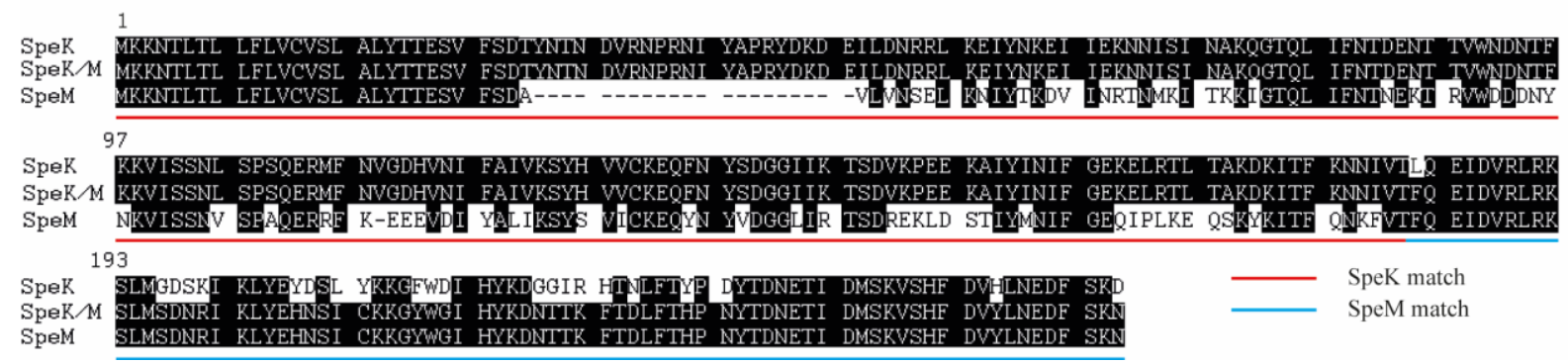

Supplementary Figure 5: Alignment of the SpeK/SpeM fusion protein to SpeK and SpeM.

Within an emm65 isolate from LIC, we identified a gene that encodes for 259 amino acids (aa) of which the first 180 aa were $100 \%$ identical to the first 180 aa of SpeK (red underlined) but the remaining 181-259 aa were $100 \%$ identical to the last 159-237 aa of SpeM (blue underlined). Black shading indicates identical aa. 

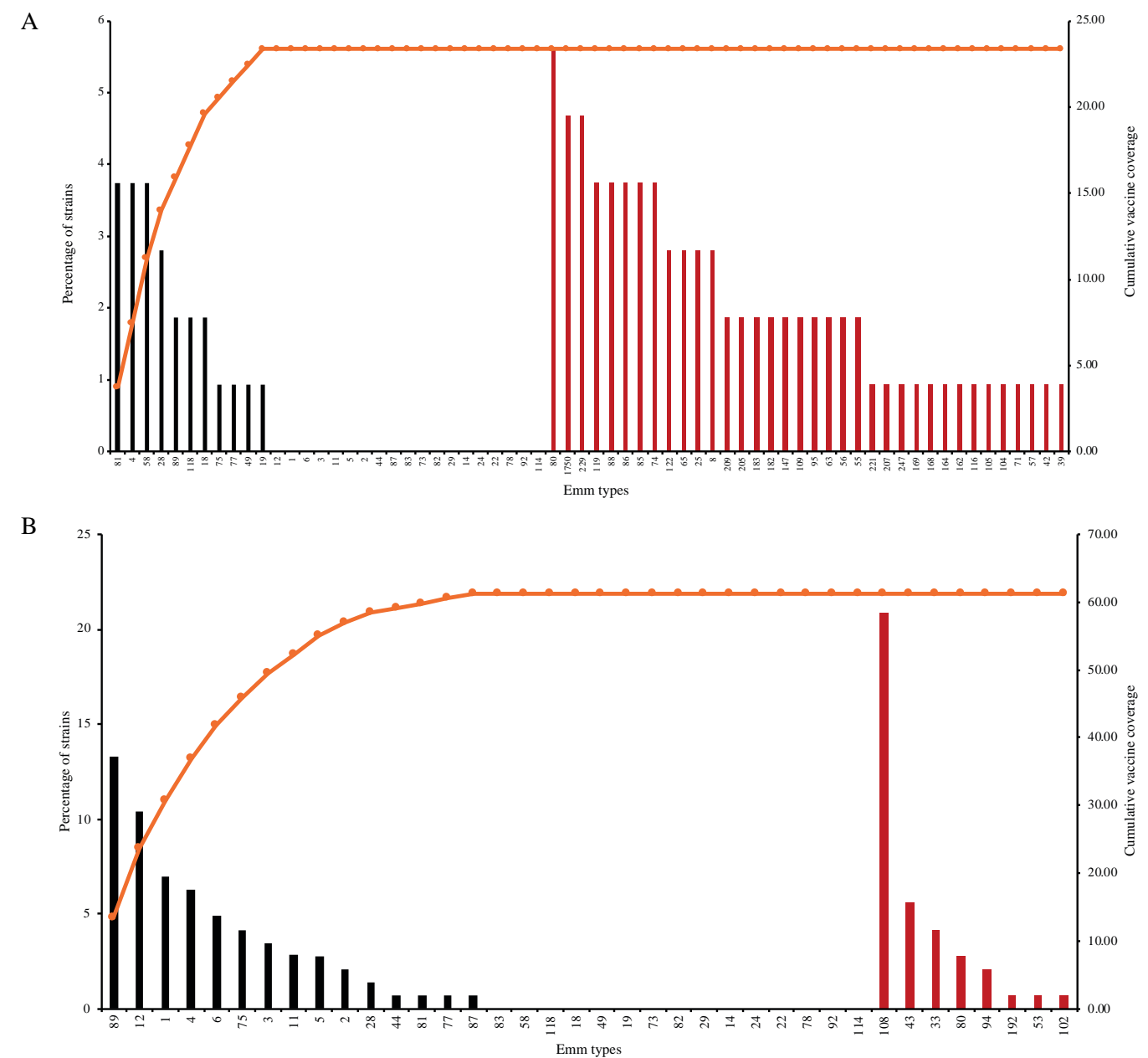

Supplementary Figure 6: Potential coverage of the $S$. pyogenes 30-valent vaccine. The percentage of (A) LIC and (B) HIC isolates of emm-types included in the 30-valent vaccine (black) and other emm-types identified in each site but not included in the vaccine (red). The cumulative vaccine coverage for each site is also shown. The emm-types without the bars are vaccine included emm-types but not seen in the dataset. 